\title{
Reducing orbital eccentricity in binary black hole simulations
}

\author{
Harald P Pfeiffer ${ }^{1}$, Duncan A Brown ${ }^{1,2}$, Lawrence E Kidder ${ }^{3}$, \\ Lee Lindblom ${ }^{1}$, Geoffrey Lovelace ${ }^{1}$ and Mark A Scheel ${ }^{1}$ \\ ${ }^{1}$ Theoretical Astrophysics, California Institute of Technology, Pasadena, CA 91125, USA \\ 2 LIGO Laboratory, California Institute of Technology, Pasadena, CA 91125, USA \\ ${ }^{3}$ Center for Radiophysics and Space Research, Cornell University, Ithaca, NY 14853, USA
}

Received 19 February 2007, in final form 17 April 2007

Published 30 May 2007

Online at stacks.iop.org/CQG/24/S59

\begin{abstract}
Binary black hole simulations starting from quasi-circular (i.e., zero radial velocity) initial data have orbits with small but nonzero orbital eccentricities. In this paper, the quasi-equilibrium initial-data method is extended to allow nonzero radial velocities to be specified in binary black hole initial data. New low-eccentricity initial data are obtained by adjusting the orbital frequency and radial velocities to minimize the orbital eccentricity, and the resulting ( $\sim 5$ orbit) evolutions are compared with those of quasi-circular initial data. Evolutions of the quasi-circular data clearly show eccentric orbits, with eccentricity that decays over time. The precise decay rate depends on the definition of eccentricity; if defined in terms of variations in the orbital frequency, the decay rate agrees well with the prediction of Peters (1964 Phys. Rev. 136 122432). The gravitational waveforms, which contain $\sim 8$ cycles in the dominant $l=m=2$ mode, are largely unaffected by the eccentricity of the quasi-circular initial data. The overlap between the dominant mode in the quasi-circular evolution and the same mode in the low-eccentricity evolution is about 0.99 .
\end{abstract}

PACS numbers: 04.25.Dm, 04.30.Db, 04.70.Bw

(Some figures in this article are in colour only in the electronic version)

\section{Introduction}

The inspiral and merger of binary black holes is one of the most promising sources for current and future generations of interferometric gravitational wave detectors such as LIGO and VIRGO $[1,2]$. The initial LIGO detectors, which are currently operating at design sensitivity, could detect binary black hole inspirals up to distances of several hundred megaparsecs. In order to take full advantage of the sensitivity of these detectors, detailed knowledge of the gravitational waveform is required. 

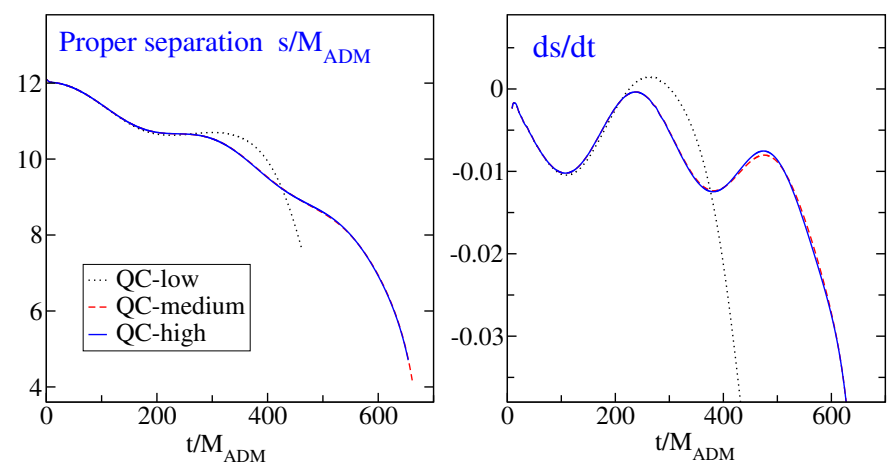

Figure 1. Evolution of quasi-circular initial data. The left panel shows the proper separation $s$ between the apparent horizons, computed at constant coordinate time along the coordinate line connecting the centres of the horizons, and the right panel shows its time derivative $\mathrm{d} s / \mathrm{d} t$. This evolution was run at three different resolutions, with the medium and high resolution tracking each other very closely through the run.

Recent breakthroughs in numerical relativity have allowed several research groups to simulate binary black hole inspirals for multiple orbits [3-7]. Because of the large computational cost of these simulations, only a small number of orbits can be followed. Therefore, it is important to begin these simulations with initial data that closely approximate a snapshot of a binary black hole system that is only a few orbits from merger. During the inspiral, the orbits of binary compact objects circularize via the emission of gravitational waves [8], so binaries formed from stellar evolution (rather than dynamical capture) are expected to have very small eccentricities by the time they enter the sensitive band of ground-based detectors. Because of this, the assumption of a quasi-circular orbit (i.e., zero radial velocity) has been widely used in the construction of binary black hole initial data [9-23]. Specifically, quasi-equilibrium data [17] and the 'QC-sequence' [24] of puncture data [25] seem to be the most popular, and both of these assume a quasi-circular orbit. However, inspiralling compact objects have a small inward radial velocity, and neglecting this velocity when constructing initial data will lead to eccentricity in the subsequent evolution, as discussed in the context of post-Newtonian theory in [26], and found numerically in [27].

The Caltech/Cornell collaboration has recently completed successful long-term simulations of inspiralling binary black holes [6] using a pseudo-spectral multi-domain method. This technique was used to evolve a particular quasi-circular quasi-equilibrium binary black hole initial data set (coordinate separation $d=20$ from table 4 of [17]). Figure 1 shows the proper separation $s$ between the horizons and the radial velocity $\mathrm{d} s / \mathrm{d} t$ as functions of time for this evolution. The rapid convergence afforded by spectral methods is apparent; the medium and high resolutions are nearly indistinguishable on the plot. Eccentricity of the orbit in the form of oscillatory variations in $s$ and $\mathrm{d} s / \mathrm{d} t$ is, unfortunately, also clearly apparent.

This notable eccentricity suggests two questions. First, how can initial data with the appropriate black hole radial velocities be constructed for non-eccentric inspirals? Second, how do evolutions of quasi-circular initial data differ from those of non-eccentric initial data? This paper addresses both questions. In section 2, we incorporate nonzero radial velocities into the quasi-equilibrium method to construct binary black hole initial data. This results in one additional parameter for equal mass initial data, the radial velocity $v_{r}$. Section 3 briefly discusses our numerical methods. Section 4 describes how we choose $v_{r}$ and the 
orbital frequency $\Omega_{0}$ for equal mass co-rotating binary black holes, and presents numerical evolutions of the resulting low-eccentricity initial data. This section also presents convergence tests of these binary black hole evolutions; we examine both convergence with respect to spatial resolution and convergence with respect to the radius of the outer boundary of the computational domain. Section 5 examines the differences between evolutions of quasicircular initial data and low-eccentricity initial data. We close with a summary and discussion of these results in section 6.

\section{Quasi-equilibrium data with nonzero radial velocity}

In this section, we extend the quasi-equilibrium approach $[14,16,17,20]$ to allow specification of nonzero radial velocities of the black holes. We proceed in three steps. First, we summarize the construction of quasi-equilibrium data using co-rotating coordinates [17,20]. Second, we show that the identical quasi-circular initial data can be obtained by solving essentially the same equations in an asymptotically inertial coordinate system; the major difference is that one must require the black holes to move on circular trajectories, rather than remaining fixed in the coordinate system. Third, we generalize from black holes moving on circular trajectories to black holes moving on inspiral trajectories.

\subsection{Overview}

We use the nomenclature of [17]; the spacetime line element is written in the usual $3+1$-form

$$
\mathrm{d} s^{2}=-\alpha^{2} \mathrm{~d} t^{2}+\gamma_{i j}\left(\mathrm{~d} x^{i}+\beta^{i} \mathrm{~d} t\right)\left(\mathrm{d} x^{j}+\beta^{j} \mathrm{~d} t\right),
$$

where $\gamma_{i j}$ is the 3-metric induced on a $t=$ constant spatial hypersurface, $\alpha$ is the lapse function and $\beta^{i}$ is the shift vector. Latin indices label spatial coordinates, and Greek indices label spacetime coordinates. The extrinsic curvature of the hypersurface is defined by

$$
K_{\mu \nu} \equiv-\gamma_{\mu}{ }^{\rho} \gamma_{\nu}{ }^{\sigma(4)} \nabla_{(\rho} n_{\sigma)},
$$

where ${ }^{(4)} \nabla$ is the spacetime derivative operator and $n_{\mu}$ is the future-pointing unit normal to the slice ${ }^{4}$. We use the extended conformal thin sandwich formalism [28, 29] to construct constraint-satisfying initial data. In this approach, the three-dimensional metric is spit into a conformal metric $\tilde{\gamma}_{i j}$ and a positive conformal factor $\psi$,

$$
\gamma_{i j}=\psi^{4} \tilde{\gamma}_{i j}
$$

and the extrinsic curvature is split into trace and trace-free parts

$$
K_{i j}=A_{i j}+\frac{1}{3} \gamma_{i j} K \text {. }
$$

The freely specifiable data consist of the conformal metric $\tilde{\gamma}_{i j}$, its time derivative $\tilde{u}_{i j} \equiv \partial_{t} \tilde{\gamma}_{i j}$ (which is taken to be trace-free), the mean curvature $K \equiv K_{i j} \gamma^{i j}$, and its time derivative $\partial_{t} K$. It follows that the trace-free part of the extrinsic curvature takes the form

$$
A_{i j}=\frac{1}{2 \alpha}\left[(\mathbb{L} \beta)_{i j}-\psi^{4} \tilde{u}_{i j}\right]=\psi^{-2} \tilde{A}_{i j}, \quad \tilde{A}_{i j}=\frac{1}{2 \tilde{\alpha}}\left[(\tilde{\mathbb{L}} \beta)_{i j}-\tilde{u}_{i j}\right],
$$

where

$$
(\mathbb{L} \beta)^{i j} \equiv 2 \nabla^{(i} \beta^{j)}-\frac{2}{3} \gamma^{i j} \nabla_{k} \beta^{k}, \quad(\tilde{\mathbb{L}} \beta)^{i j} \equiv 2 \tilde{\nabla}^{(i} \beta^{j)}-\frac{2}{3} \tilde{\gamma}^{i j} \tilde{\nabla}_{k} \beta^{k} .
$$

The symbols $(\mathbb{L} \beta)^{i j}$ and $(\tilde{\mathbb{L}} \beta)^{i j}$ represent the conformal Killing operators in physical and conformal space, respectively, and are related by $(\mathbb{L} \beta)^{i j}=\psi^{-4}(\tilde{\mathbb{L}} \beta)^{i j}$. Indices

4 Since $K_{\mu \nu}$ is a spatial tensor, $K_{\mu \nu} n^{v}=0$, its spatial components $K_{i j}$ carry all its information. Almost all tensors in this paper are spatial, and we use spatial indices here whenever possible. 
on conformal tensors are raised and lowered with the conformal metric, for example, $(\tilde{\mathbb{L}} \beta)_{i j} \equiv \tilde{\gamma}_{i k} \tilde{\gamma}_{j l}(\tilde{\mathbb{L}} \beta)^{k l}=\psi^{-4}(\mathbb{L} \beta)_{i j}$. Furthermore, $\nabla$ and $\tilde{\nabla}$ denote the physical and conformal spatial covariant derivative operators, and the conformal lapse is defined by $\alpha=\psi^{6} \tilde{\alpha}$. Inverting equation (5) yields

$$
\tilde{u}_{i j}=\partial_{t} \tilde{\gamma}_{i j}=-2 \tilde{\alpha} \tilde{A}_{i j}+(\tilde{\mathbb{L}} \beta)_{i j}
$$

Substituting these relations into the constraint equations and into the evolution equation for the extrinsic curvature, one arrives at a system of five elliptic equations, often referred to as the extended conformal thin sandwich (XCTS) equations

$$
\begin{aligned}
& \tilde{\nabla}^{2} \psi-\frac{1}{8} \tilde{R} \psi-\frac{1}{12} K^{2} \psi^{5}+\frac{1}{8} \psi^{-7} \tilde{A}^{i j} \tilde{A}_{i j}=0, \\
& \tilde{\nabla}_{j}\left(\frac{1}{2 \tilde{\alpha}}(\tilde{\mathbb{L}} \beta)^{i j}\right)-\frac{2}{3} \psi^{6} \tilde{\nabla}^{i} K-\tilde{\nabla}_{j}\left(\frac{1}{2 \tilde{\alpha}} \tilde{u}^{i j}\right)=0, \\
& \tilde{\nabla}^{2}\left(\tilde{\alpha} \psi^{7}\right)-\left(\tilde{\alpha} \psi^{7}\right)\left[\frac{\tilde{R}}{8}+\frac{5}{12} K^{4} \psi^{4}+\frac{7}{8} \psi^{-8} \tilde{A}^{i j} \tilde{A}_{i j}\right]=-\psi^{5}\left(\partial_{t} K-\beta^{k} \partial_{k} K\right) .
\end{aligned}
$$

Here $\tilde{R}$ denotes the trace of the Ricci tensor of $\tilde{\gamma}_{i j}$ and we have specialized to the vacuum case. These equations are to be solved for $\psi, \tilde{\alpha}$ and $\beta^{i}$; given a solution, the physical initial data $\left(\gamma_{i j}, K_{i j}\right)$ are obtained from equations (3)-(5).

Note that a solution of the XCTS equations includes a shift vector $\beta^{i}$ and a lapse function $\alpha=\psi^{6} \tilde{\alpha}$. If these values of lapse and shift are used in an evolution of the constructed initial data, then the time derivative of the mean curvature will initially equal the freely specifiable quantity $\partial_{t} K$, and the trace-free part of the time derivative of the metric will initially equal $\psi^{4} \tilde{u}_{i j}$. Thus, the free data of the XCTS equations allow direct control of certain time derivatives in the evolution of the initial data.

The next step is to choose the free data that correspond to the desired physical configuration. The quasi-equilibrium quasi-circular orbit method of constructing binary black holes $[17,20]$ (see also $[11,12,14]$ ) provides a framework for many of these choices. This method is based on the fact that the inspiral time scale for a binary compact object is much larger than the orbital time scale, so that time derivatives should be very small in the co-rotating coordinate system. Furthermore, the black holes should be in equilibrium, which provides conditions on the expansion $\theta$ and shear $\sigma_{i j}$ of the outgoing null geodesics passing through the horizon. The complete set of physically motivated choices for the free data within the quasi-equilibrium method is

$$
\begin{aligned}
& \tilde{u}_{i j}=0, \\
& \partial_{t} K=0, \\
& \psi \rightarrow 1, \quad \alpha \rightarrow 1, \quad \text { as } r \rightarrow \infty, \\
& \beta^{i} \rightarrow\left(\Omega_{0} \times \mathbf{r}\right)^{i}, \\
& \partial_{t} \text { is tangent to } \mathcal{S}_{\mathrm{AH}}, \\
& \theta=0 \quad \text { on } \mathcal{S}, \\
& \sigma_{i j}=0 \text { on } \mathcal{S},
\end{aligned}
$$

where $\mathcal{S}$ denotes the location of the apparent horizons in the initial data surface, and $\mathcal{S}_{A H}$ is the world tube of the apparent horizon obtained by evolving the initial data with lapse $\alpha$ and 
shift $\beta^{i}$. The first two conditions are the assumptions that the time derivatives are small. The boundary conditions in equations $(9 c)$ and $(9 d)$ enforce asymptotic flatness and co-rotation. The orbital frequency $\Omega_{0}$ entering equation $(9 d)$ can be chosen by the effective potential method [9] or the Komar-mass ansatz [11], with similar results [20].

To discuss the remaining conditions, we need to introduce a few additional geometrical quantities. Denote by $s^{i}$ and $\tilde{s}^{i}$ the physical and conformal outward-pointing spatial unit normals to $\mathcal{S}$. They obey the relations

$$
s^{i} s^{j} \gamma_{i j}=1, \quad \tilde{s}^{i} \tilde{s}^{j} \tilde{\gamma}_{i j}=1, \quad s^{i}=\psi^{-2} \tilde{s}^{i} .
$$

Then introduce the induced metric on $\mathcal{S}$ in physical and conformal space by $h_{i j}=\gamma_{i j}-s_{i} s_{j}$ and $\tilde{h}_{i j}=\tilde{\gamma}_{i j}-\tilde{s}_{i} \tilde{s}_{j}$, respectively. Because $n_{\mu} s^{\mu}=0$, the spacetime components of the unit normal are given by $s^{\mu}=\left[0, s^{i}\right]$. The outward-pointing null normal to $\mathcal{S}$ can then be written as

$$
k^{\mu}=\frac{1}{\sqrt{2}}\left(n^{\mu}+s^{\mu}\right)
$$

Equation $(9 e)$ simply means that the apparent horizon is initially at rest when the initial data are evolved in the co-rotating coordinate system. It implies that the shift must take the form

$$
\beta^{i}=\alpha s^{i}+\beta_{\|}^{i} \quad \text { on } \mathcal{S}
$$

where $\beta_{\|}^{i}$ is tangent to $\mathcal{S}$. Equation $(9 f)$ ensures that $\mathcal{S}$ is an apparent horizon, and implies a boundary condition on the conformal factor

$$
\tilde{s}^{k} \partial_{k} \psi=-\frac{\psi^{-3}}{8 \tilde{\alpha}} \tilde{s}^{i} \tilde{s}^{j}\left[(\tilde{\mathbb{L}} \beta)_{i j}-\tilde{u}_{i j}\right]-\frac{\psi}{4} \tilde{h}^{i j} \tilde{\nabla}_{i} \tilde{s}_{j}+\frac{1}{6} K \psi^{3} .
$$

Finally, equation $(9 g)$ — which forces the apparent horizon to be in equilibrium—restricts $\beta_{\|}^{i}$ to be a conformal Killing vector within the surface $\mathcal{S}$,

$$
\left(\tilde{\mathbb{L}}_{\mathcal{S}} \beta_{\|}\right)^{i j} \equiv 2 \tilde{D}^{(i} \beta_{\|}^{j)}-\tilde{h}^{i j} \tilde{D}_{k} \beta_{\|}^{k}=0,
$$

where $\tilde{D}_{i}$ is the covariant derivative compatible with $\tilde{h}_{i j}$. As discussed in detail in [17, 20], $\beta_{\|}^{i}$ controls the spin of the black holes in addition to the spin required for co-rotation.

Quasi-equilibrium considerations have now led us to choices for half of the free data $\left(\tilde{u}_{i j}\right.$ and $\partial_{t} K$ ) for the XCTS equations, and for all boundary conditions except a lapse boundary condition on the horizon $\mathcal{S}$. As argued in [17], equations $(9 a)-(9 e)$ are compatible with any spin of the black holes, with any choice of boundary conditions for the lapse on $\mathcal{S}$, and with any choice of $\tilde{\gamma}_{i j}$ and $K$. For concreteness, we choose

$$
\begin{aligned}
& \tilde{\gamma}_{i j}=f_{i j}, \\
& K=0, \\
& \partial_{r}(\alpha \psi)=0 \text { on } \mathcal{S},
\end{aligned}
$$

where $f_{i j}$ is the Euclidean metric. The last two conditions, equations $(15 b)$ and $(15 c)$, are gauge choices [17]. The choice of the conformal metric, however, does influence the physical gravitational radiation degrees of freedom of the system. Since a black hole binary is not conformally flat at second post-Newtonian order [30], our simple choice of conformal flatness, equation (15a), is probably responsible for the initial burst of unphysical gravitational radiation found in the evolution of these initial data. 


\subsection{Initial data in an asymptotically inertial frame}

It is possible to re-formulate the quasi-equilibrium method in asymptotically inertial coordinates in such a way that identical physical initial data are obtained. To do so, we solve the XCTS equations $(8 a)-(8 c)$ with the same choices for the free data and boundary conditions, except that equations $(9 d)$ and $(9 e)$ are replaced by

$$
\begin{aligned}
& \beta^{i} \rightarrow 0 \quad \text { as } r \rightarrow \infty, \\
& \partial_{t}+\xi_{\text {rot }}^{i} \partial_{i} \text { is tangent to } \mathcal{S}_{\mathrm{AH}}, \quad \text { where } \quad \xi_{\text {rot }}^{i}=\left(\boldsymbol{\Omega}_{\mathbf{0}} \times \mathbf{r}\right)^{i} .
\end{aligned}
$$

The second condition implies that the apparent horizons move initially with velocity $\xi_{\text {rot }}^{i}$, i.e. tangent to circular orbit trajectories.

Let $\left(\psi_{\mathrm{co}}, \beta_{\mathrm{co}}^{i}, \alpha_{\mathrm{co}}\right)$ be the solution to the XCTS equations in the co-rotating coordinates. We show in appendix A that the solution in the asymptotically inertial coordinates is $\left(\psi, \beta^{i}, \alpha\right)=\left(\psi_{\mathrm{co}}, \beta_{\mathrm{co}}^{i}-\xi_{\text {rot }}^{i}, \alpha_{\mathrm{co}}\right)$, and that this solution leads to the same physical metric $\gamma_{i j}$ and extrinsic curvature $K_{i j}$ as the original solution in co-rotating coordinates. The proof of this relies on two observations. First, the shift enters the XCTS equations and the boundary conditions (almost) solely through the conformal Killing operator, $(\tilde{\mathbb{L}} \beta)^{i j}$, and second, $\xi_{\text {rot }}^{i}$ is a conformal Killing vector, $\left(\tilde{\mathbb{L}} \xi_{\text {rot }}\right)^{i j}=0$, for the conformally flat case considered here. Hence the term $-\xi_{\text {rot }}^{i}$ that is added to $\beta_{\text {co }}^{i}$ drops out of the equations.

In appendix $\mathrm{A}$, we also show that equation $(16 b)$ and the shear condition equation $(9 g)$ require the shift on the inner boundary $\mathcal{S}$ to take the form

$$
\beta^{i}=\alpha s^{i}-\xi_{\text {rot }}^{i}+\zeta^{i} \text { on } \mathcal{S}
$$

where $\zeta^{i}$ is a vector that must be tangent to $\mathcal{S}\left(\zeta^{i} s_{i}=0\right)$ and must be a conformal Killing vector within the surface $\mathcal{S}$ :

$$
\sigma_{i j}=0 \quad \Leftrightarrow \quad 0=\left(\tilde{\mathbb{L}}_{\mathcal{S}} \zeta\right)^{i j}
$$

Comparing equation (17) with equation (12), we see that the vector $\zeta^{i}$ plays the role of $\beta_{\|}^{i}$ in the earlier treatment; choosing it as a rotation within $\mathcal{S}$ will impart additional spin to the black holes in addition to co-rotation, as described in detail in [20]. Note that at large radii the comoving shift $\beta_{\mathrm{co}}^{i}$ is a pure rotation, since $\beta_{\mathrm{co}}^{i} \rightarrow \xi_{\mathrm{rot}}^{i}$ (equation (16a)) and $\nabla^{j} \xi_{\mathrm{rot}}^{i}$ is antisymmetric (equation (16b)).

\subsection{Initial data with nonzero radial velocity}

After rewriting the standard quasi-equilibrium method in an asymptotically inertial frame, it is straightforward to incorporate nonzero initial radial velocities for the black holes. As discussed in section 2.2, quasi-circular initial data can be generated by specifying that the horizons move initially on circles in an asymptotically inertial coordinate system. This is accomplished by the shift boundary conditions in equations (16a) and (16b). We include initial radial velocities simply by requiring the black holes to move initially on inspiral rather than circular trajectories.

Consider the problem of giving a black hole located at a distance $r_{0}$ from the origin an initial radial velocity $v_{r}$. This can easily be accomplished by replacing the boundary conditions in equations $(16 a)$ and (16b) with

$$
\begin{aligned}
& \beta^{i} \rightarrow 0 \quad \text { as } r \rightarrow \infty, \\
& \partial_{t}+\xi_{\text {insp }}^{i} \partial_{i} \text { is tangent to } \mathcal{S}_{\mathrm{AH}}, \quad \text { where } \quad \xi_{\text {insp }}^{i} \equiv\left(\boldsymbol{\Omega}_{\mathbf{0}} \times \mathbf{r}\right)^{i}+v_{r} \frac{r^{i}}{r_{0}} .
\end{aligned}
$$


As before, we place the centre of rotation at the origin of the coordinate system. Note that $\xi_{\text {insp }}^{i}$ is still a conformal Killing vector, $\left(\tilde{\mathbb{L}} \xi_{\text {insp }}\right)^{i j}=0$, for the conformally flat case considered here. Therefore, the analysis in appendix A of the boundary conditions in equations (16b) and $(9 g)$ also applies to equations $(19 b)$ and $(9 g)$, and so we find that the inner shift boundary condition must be of the form

$$
\beta^{i}=\alpha s^{i}-\xi_{\text {insp }}^{i}+\zeta^{i}, \quad \text { on } \mathcal{S}
$$

where $\zeta^{i}$ is a conformal Killing vector within $\mathcal{S}$.

The boundary conditions in equations $(19 a)$ and $(19 b)$ depend on two parameters, the orbital frequency $\Omega_{0}$ and a radial velocity $v_{r}$ (or, more precisely, an overall expansion factor $v_{r} / r_{0}$, reminiscent of the Hubble constant). For unequal mass binary systems the needed radial velocities for each hole would be different, but the needed expansion factors, $v_{r} / r_{0}$, are expected to be the same for the two holes.

The changes discussed in section 2.2 are superficially similar to the changes discussed in section 2.3, yet the former amounts to a mere coordinate transformation while the latter produces different physical initial data. This can be understood by noting that the change from co-rotating coordinates (equations $(9 d)$ and $(9 e)$ ) to inertial coordinates (equations $(16 a)$ and $(16 b))$ is accomplished by adding the same conformal Killing vector field $\xi_{\text {rot }}^{i}$ to the shift at both inner and outer boundaries, but the change from equations $(9 d)$ and $(9 e)$ to initial data with nonzero radial velocity (equations $(19 a)$ and $(19 b)$ ) is accomplished by adding different conformal Killing fields to the shift on different boundaries: $\xi_{\text {rot }}^{i}$ at the outer boundary and $\xi_{\text {insp }}^{i}$ at the inner boundaries. Only in the former case can the change be expressed as a global transformation of the shift of the form $\beta^{i} \rightarrow \beta^{i}+\xi_{\text {rot }}^{i}$.

\section{Numerical methods}

The initial value equations are solved with the pseudo-spectral elliptic solver described in [31]. This elliptic solver has been updated to share the more advanced infrastructure of our evolution code and is now capable of handling cylindrical subdomains. This increases its efficiency by about a factor of 3 over the results described in [31] for binary black hole initial data.

The Einstein evolution equations are solved with the pseudo-spectral evolution code described in [6]. This code evolves a first-order representation [32] of the generalized harmonic system [33, 34]. We use outer boundary conditions [32] designed to prevent the influx of unphysical constraint violations and undesired incoming gravitational radiation, while allowing the outgoing gravitational radiation to pass freely through the boundary. The code uses a fairly complicated domain decomposition. Each black hole is surrounded by three concentric spherical shells, with the inner boundary of the inner shell just inside the horizon. The inner shells overlap a structure of 24 touching cylinders, which in turn overlap a set of outer spherical shells — centred at the origin — which extend to large outer radius. Outer boundary conditions are imposed only on the outer surface of the largest outer spherical shell. We vary the location of the outer boundary by adding more shells at the outer edge. Since all outer shells have the same angular resolution, the cost of placing the outer boundary farther away (at full resolution) increases only linearly with the radius of the boundary. Some of the details of the domain decompositions used for the simulations presented here are given in table 1 . 
Table 1. Summary of evolutions presented in this paper. The labels 'QC', 'E' and 'F' refer to the different initial data sets, with numerical suffix (E1, E2 etc) denoting different values of the initial outer boundary radius of the evolutions, $R_{\text {outer }}$.

\begin{tabular}{|c|c|c|c|c|c|c|}
\hline \multirow[b]{2}{*}{ Label } & \multirow[b]{2}{*}{ Initial data } & \multirow[b]{2}{*}{$\frac{R_{\text {outer }}}{M_{\mathrm{ADM}}}$} & \multirow{2}{*}{$\begin{array}{l}\text { \# Outer } \\
\text { shells }\end{array}$} & \multicolumn{3}{|c|}{ Approx. \# of points } \\
\hline & & & & Low & Medium & High \\
\hline QC & $\begin{array}{l}M_{\mathrm{ADM}} \Omega_{0}=0.029792, v_{r}=0.0 \\
J_{\mathrm{ADM}} / M_{\mathrm{ADM}}^{2}=0.98549 \\
M_{\mathrm{irr}} / M_{\mathrm{ADM}}=0.50535\end{array}$ & 133 & 8 & $52^{3}$ & $64^{3}$ & $76^{3}$ \\
\hline E1 & $M_{\mathrm{ADM}} \Omega_{0}=0.029961, v_{r}=-0.0017$ & 171 & 10 & $59^{3}$ & $66^{3}$ & $74^{3}$ \\
\hline E2 & $\begin{array}{l}J_{\mathrm{ADM}} / M_{\mathrm{ADM}}^{2}=0.99172 \\
M_{\mathrm{irr}} / M_{\mathrm{ADM}}=0.50524\end{array}$ & 293 & 18 & $64^{3}$ & $72^{3}$ & $81^{3}$ \\
\hline F1 & $M_{\mathrm{ADM}} \Omega_{0}=0.029963, v_{r}=-0.0015$ & 133 & 8 & $52^{3}$ & $64^{3}$ & $76^{3}$ \\
\hline $\mathrm{F} 2$ & $J_{\mathrm{ADM}} / M_{\mathrm{ADM}}^{2}=0.99164$ & 190 & 12 & $55^{3}$ & $66^{3}$ & $78^{3}$ \\
\hline F3 & $M_{\mathrm{irr}} / M_{\mathrm{ADM}}=0.50525$ & 419 & 28 & $62^{3}$ & $74^{3}$ & $87^{3}$ \\
\hline
\end{tabular}

\section{Choice of orbital frequency and radial velocity}

We now describe how to construct binary black hole initial data sets with low orbital eccentricity. This is done by tuning the freely adjustable orbital parameters $\Omega_{0}$ and $v_{r}$ iteratively to reduce the eccentricity of the inspiral trajectories. For each iteration, we choose trial orbital parameters $\Omega_{0}$ and $v_{r}$, evolve the corresponding initial data, analyse the resulting trajectories of the black holes and update the orbital parameters to reduce any oscillatory behaviour in quantities such as the coordinate separation of the black holes $d(t)$, the proper separation between the horizons $s(t)$, or the orbital frequency $\omega(t)$. All of these quantities (and many others) exhibit similar oscillatory behaviour; we choose $d(t)$ as our primary diagnostic during the tuning process because it is most easily accessible during the evolutions.

To make this procedure quite explicit, we begin by evolving quasi-circular initial data for about two orbits. Then we measure the time derivative of the measured coordinate separation of the holes $\dot{d}(t)$ (in the asymptotic inertial coordinates used in our code [6]) as illustrated for example in figure 3 . We fit this measured $\dot{d}(t)$ to a function of the form

$$
\dot{d}(t)=A_{0}+A_{1} t+B \sin (\omega t+\varphi),
$$

where $A_{0}, A_{1}, B, \omega$ and $\varphi$ are constants determined by the fit. The $A_{0}+A_{1} t$ part of the solution represents the smooth inspiral, while the $B \sin (\omega t+\varphi)$ part represents the unwanted oscillations due to the eccentricity of the orbit. For a nearly circular Newtonian orbit, $B$ is related to the eccentricity $e$ of the orbit by $e=B / \omega d$. So reducing the orbital eccentricity is equivalent to reducing $B$. The values of the orbital parameters $\Omega_{0}$ and $v_{r}$ are now adjusted iteratively to make the coefficient $B$ in this fit as small as desired. After each adjustment of $\Omega_{0}$ and $v_{r}$, the initial value equations described in section 2 (in particular, using the boundary condition (19b) which depends on $\Omega_{0}$ and $v_{r}$ ) are solved completely (to the level of numerical truncation error).

For this paper, our goal is to reduce $B$, and hence the orbital eccentricity, by about a factor of 10 compared to quasi-circular initial data. This level of reduction is sufficient to allow us to evaluate the significance of the orbital eccentricity inherent in quasi-circular initial data. A variety of methods could be used to find orbital parameters that make $B$ small. One possibility is simply to evaluate $B\left(\Omega_{0}, v_{r}\right)$ numerically as described above, and then to use 
standard numerical methods to solve the equation $B\left(\Omega_{0}, v_{r}\right)=0$. Since our goal in this paper is to reduce $B$ by about a factor of 10 , simple bisection root finding methods are sufficient.

A more efficient method is to use our knowledge of the behaviour of nearly circular orbits to make informed estimates of the needed adjustments in the orbital parameters. Evaluating the fit equation (21) at the initial time $t=0$, we see that the ellipticity-related component $B \sin (\omega t+\varphi)$ contributes $B \sin (\varphi) / 2$ to the radial velocity of each hole and $B \omega \cos (\varphi) / 2$ to its radial acceleration. (The factor $1 / 2$ arises because $d$ measures the distance between the holes.) For a Newtonian binary, this eccentricity-induced radial velocity can be completely removed by changing the initial radial velocity by

$$
\delta v_{r}=-\frac{B \sin (\varphi)}{2} .
$$

Furthermore, changing the orbital frequency $\Omega_{0}$ by a small amount $\delta \Omega_{0}$ changes the radial acceleration of each black hole by the amount $\Omega_{0} \delta \Omega_{0} d_{0}$, where $d_{0}=d(0)$ is the initial separation of the holes. Thus the change $\delta \Omega_{0}$ needed to remove the eccentricity-induced initial radial acceleration, $B \omega \cos (\varphi) / 2$, is

$$
\delta \Omega_{0}=-\frac{B \omega \cos (\varphi)}{2 d_{0} \Omega_{0}} \approx-\frac{B \cos (\varphi)}{2 d_{0}} .
$$

Equations (22) and (23) still hold approximately for relativistic binaries. We have found that simultaneously adjusting $v_{r}$ and $\Omega_{0}$ by equations (22) and (23) typically reduces $B$ by about a factor of 10 .

The smallest eccentricity data set produced here (by the simple bisection method described above) is labelled ' $F$ ', and the data from the next to last iteration of this method are labelled ' $E$ '. These initial data sets, together with the quasi-circular data labelled 'QC' were evolved with multiple numerical resolutions and with multiple outer boundary locations; table 1 summarizes these evolutions. The orbital frequency used in the final evolution is only $0.6 \%$ larger than the value of $\Omega_{0}$ used in the quasi-circular case. As expected, this change is comparable to the magnitude of the radial velocity $v_{r}$ in the low-eccentricity case. The smallness of these quantities shows that the quasi-circular approximation is quite good.

Figure 2 shows the orbital phase (as measured by the coordinate locations of the centres of the apparent horizons) for the evolutions of quasi-circular initial data, QC, and the least eccentric initial data, F1, F2 and F3. (The numerical suffix, F1, F2, etc, denotes simulations with different values of the outer boundary radius as defined in table 1.) These evolutions proceed for about five orbits and then crash shortly before the black holes merge. The upper left inset shows differences between the orbital phase computed with different resolutions for the QC and the F2 runs. The phase difference between the high- and low-resolution runs is $\lesssim 0.35$ radians, which is a good estimate of the error in the low-resolution run. The phase difference between the medium and high-resolution runs drops to $\approx 0.02$ radians, which can be taken as the error in the medium resolution run. Between low and medium resolutions, the error drops by about a factor of 20. Assuming exponential convergence, the error of the high-resolution run should be smaller by yet another factor of $\sim 20$, i.e. $\lesssim 0.001$ radians. The lower right inset in figure 2 shows phase differences between evolutions of the same initial data, but run with different outer boundary radii. These differences are small, so we do not expect the influence of the outer boundary on our results to be significant. Our analysis in section 5 is based mostly on comparisons between the high-resolution QC and F2 runs.

Figure 3 illustrates the radial velocities (determined from the time derivatives of both the coordinate and the intra-horizon proper separations) for the quasi-circular run QC and for the two low-eccentricity runs $\mathrm{E}$ and F. Orbital eccentricity causes periodic oscillations in these curves; the amplitudes of these oscillations are clearly much smaller in runs $\mathrm{E}$ and 


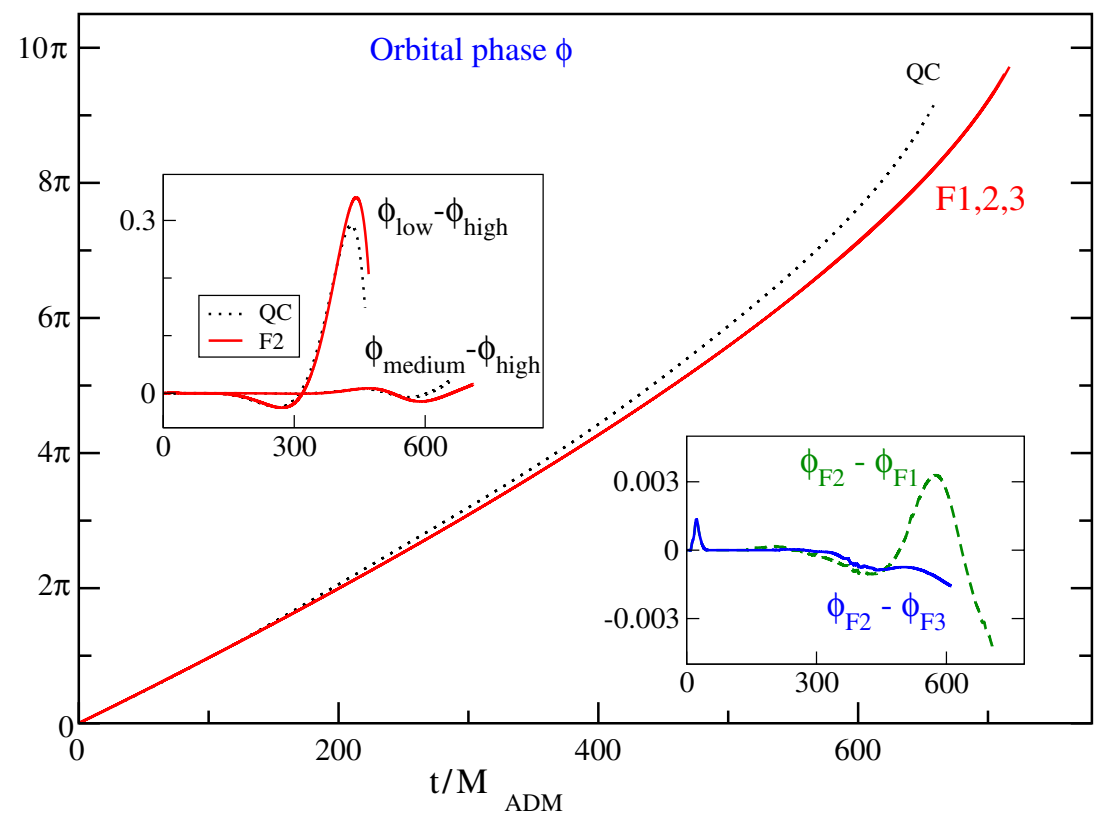

Figure 2. Evolution of the orbital phase. The main panel shows the phase of the trajectories of the centres of the apparent horizons as a function of time for the quasi-circular (dotted curves) and low-eccentricity (solid curves) initial data. The top left inset shows the phase differences between different resolution runs, which decrease at higher resolutions. The lower right inset shows the difference in the orbital phase between evolutions with different outer boundary locations.
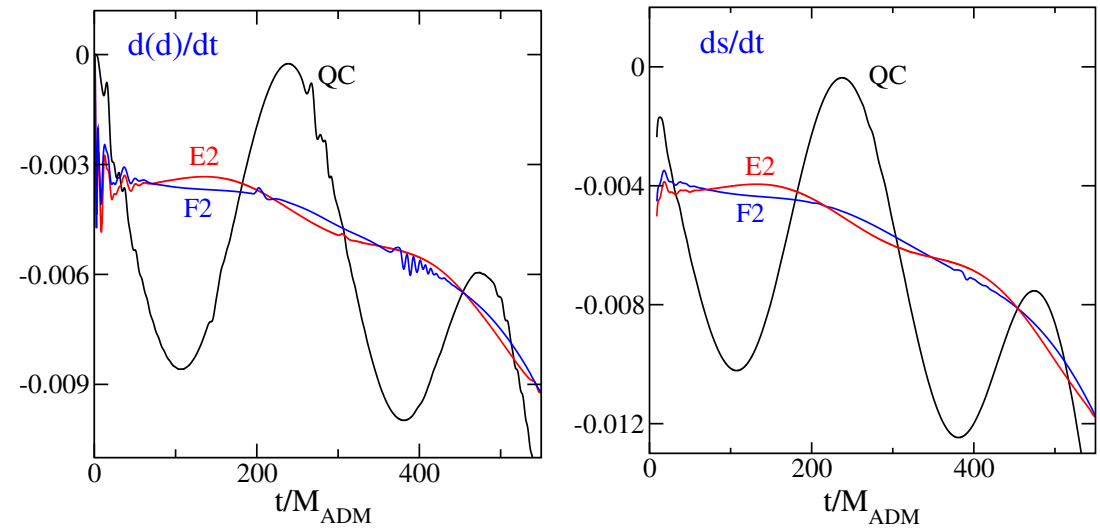

Figure 3. Radial velocity during evolutions of quasi-circular and low-eccentricity initial data. The left panel shows the coordinate velocity $\dot{d}(t)$, the right panel shows the velocity determined from the intra-horizon proper separation $\dot{s}(t)$.

$\mathrm{F}$ than in run QC. By fitting the proper separation speed $\mathrm{d} s / \mathrm{d} t$ to a linear function plus sinusoid, $\mathrm{d} s / \mathrm{d} t=A_{0}+A_{1} t+B \sin (\omega t+\varphi)$, the approximate amplitude of the oscillations can be estimated. We find $B_{\mathrm{QC}} \approx 5.5 \times 10^{-3}, B_{\mathrm{E}} \approx 5.8 \times 10^{-4}$, and $B_{\mathrm{F}} \approx 4.1 \times 10^{-4}$. This confirms that we have succeeded in our goal of reducing the oscillations by an order of magnitude. These fits are not very accurate because the fit must cover at least one period of the oscillations, and significant orbital evolution occurs during this time. If we vary the 


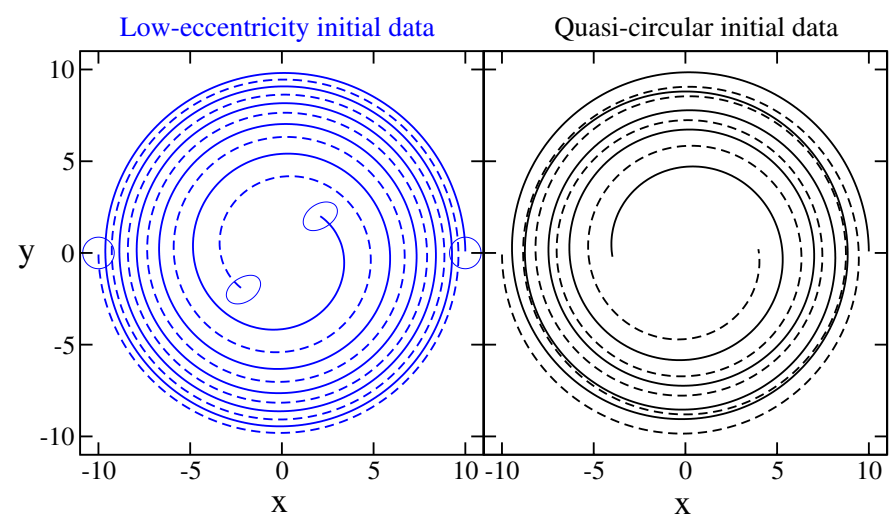

Figure 4. Trajectories of the centre of the apparent horizons in asymptotically inertial coordinates for the runs E1 (left plot) and QC (right plot). The solid-dashed lines distinguish the two black holes; the circles and ellipsoids in the left panel denote the location of the apparent horizon at the beginning and end of the evolution.

fit interval $40<t / M_{\mathrm{ADM}}<T$ by choosing $T$ between 300 and 450 , the quoted amplitudes $A_{\mathrm{QC}, \mathrm{E}, \mathrm{F}}$ change at about the $10 \%$ level.

The coordinate separation $\mathrm{d}(d) / \mathrm{d} t$ shows some noise at early times as the binary system equilibrates and an initial burst of 'junk' gravitational radiation travels outward. There are also short-lived, high-frequency features apparent in figure 3 at intermediate times. The earlier feature occurs at $t / M_{\mathrm{ADM}} \sim 140$ for the $\mathrm{QC}$ run, $t / M_{\mathrm{ADM}} \sim 200$ for $\mathrm{F} 2$, and $t / M_{\mathrm{ADM}} \sim 300$ for E2; these times coincide with the light-crossing time to the outer boundary. We believe that this early feature is caused by a small mismatch between the initial data and the outer boundary conditions used by the evolution code; this mismatch produces a pulse that propagates inward from the outer boundary starting at $t=0$. A later (and larger) feature occurs at $t / M_{\mathrm{ADM}} \sim 280$ for the $\mathrm{QC}$ run, $t / M_{\mathrm{ADM}} \sim 400$ for $\mathrm{F} 2$, and at $t / M_{\mathrm{ADM}} \sim 600$ (off the scale of figure 3 ) for E2. This latter feature occurs at twice the light-crossing time, and is caused by reflection of the initial 'junk' gravitational radiation burst off the outer boundary. The outer boundary conditions used in this paper perform well for the physical gravitational-wave degrees of freedom [32], but comparatively poorly for the gauge degrees of freedom (as demonstrated in recent tests [35]). These results plus the observation that the high-frequency features in figure 3 are greatly diminished in less gauge-dependent quantities like $\mathrm{d} s / \mathrm{d} t$ suggest that these features may be caused by perturbations in the gauge or coordinate degrees of freedom of the system.

Figure 4 shows the orbital trajectories of the centres of the black holes during evolutions of the low-eccentricity initial data E, ${ }^{5}$ and the quasi-circular initial data QC. The low-eccentricity run forms a smooth spiral with no apparent distortion. In contrast, the evolution starting from quasi-circular initial data has clearly visible irregularities.

\section{Comparing quasi-circular and low-eccentricity initial data}

Figures 3 and 4 show clearly that evolutions of the quasi-circular initial data, QC, are not the same as those of the low-eccentricity initial data, F. In this section, we characterize and quantify these differences in more detail.

5 We plot the evolution E1 because it was pushed somewhat closer to merger than the F runs; the trajectories of the E runs are indistinguishable from those of the F runs on the scale of this figure. 

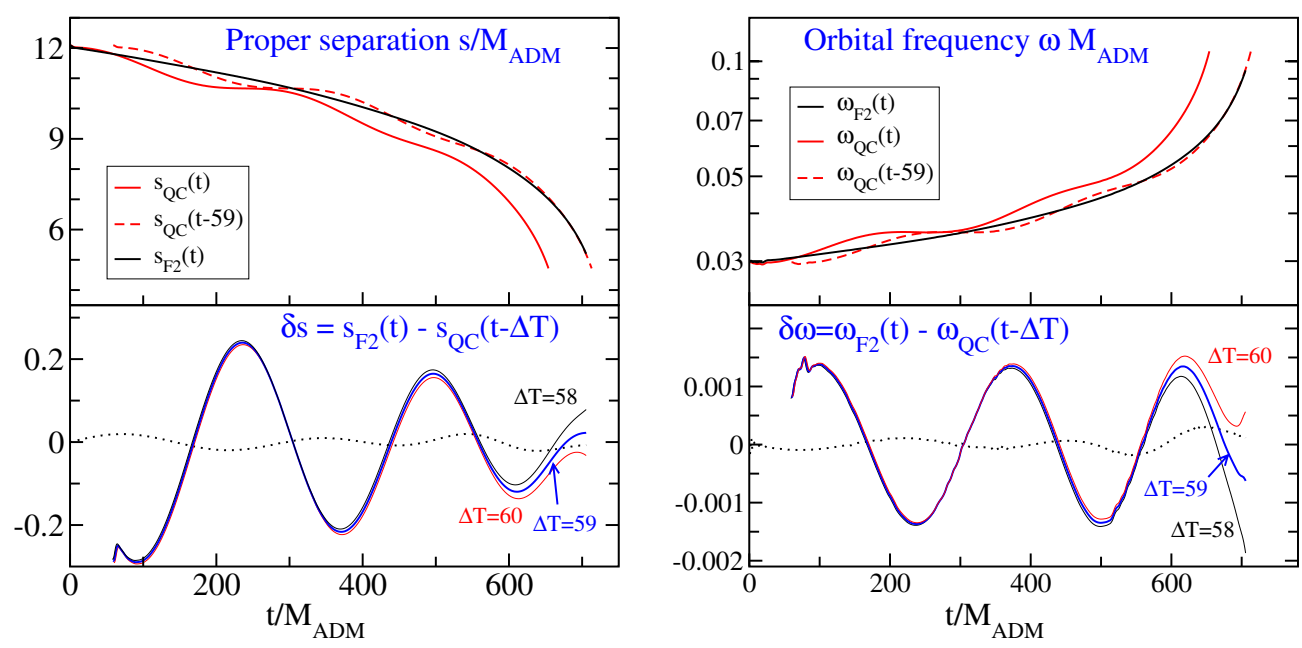

Figure 5. Proper separation (left) and orbital frequency (right) for evolutions of the QC and F initial data. The lower panels show the differences between the time-shifted QC and the F2 runs. The dotted lines in the lower panels show the differences between the E1 and F2 runs, providing an estimate of the remaining eccentricity in the F2 run.

\subsection{Time shift}

The black holes approach each other more quickly in the QC run, with the time of coalescence appearing to be about $60 \mathrm{M}_{\mathrm{ADM}}$ earlier than in the F2 run. Figure 2, for example, shows that the orbital phase increases more quickly during the QC run, with a late time phase difference of about $\pi$ (almost a full gravitational wave cycle) compared to the F2 run. Similar differences are also seen in the graphs of the proper separation and orbital frequency shown in the upper panels of figure 5 .

We find that most of the difference between the QC and F runs is just a simple coordinate time shift. The dashed lines in the upper panels of figure 5 represent the QC evolution shifted by $\Delta T=59 M_{\mathrm{ADM}}$. With this time shift, the QC evolution oscillates around the low-eccentricity F2 run. Therefore, the apparent earlier merger time of the QC run is just a consequence of the fact that coordinate time $t=0$ in the QC run represents a later stage in the inspiral than it does in the F2 evolution. The QC and F2 runs were started with the same spatial coordinate separation at $t=0$; however, this point is the apocentre of the slightly eccentric QC orbit, so the point in the F2 run with the same phase (measured from merger) has smaller separation.

The lower left panel of figure 5 shows the proper separation difference, $\delta s=s_{\mathrm{F}}(t)-$ $s_{\mathrm{QC}}(t-\Delta T)$, which emphasizes the oscillations of the QC evolution around the $\mathrm{F} 2$ orbit. These differences are plotted for three different time shifts $\Delta T$. The right panels of figure 5 present information about the orbital angular frequency $\omega$ as determined from the coordinate locations of the centres of the apparent horizons. The upper right panel shows $\omega$ for evolutions of QC and F2 initial data. Time shifting the QC run by the same $\Delta T=59 M_{\mathrm{ADM}}$ also lines up the frequency curves very well. The lower right plot shows the difference in orbital frequency between the F2 run and the time-shifted QC run, $\delta \omega=\omega_{\mathrm{F}}(t)-\omega_{\mathrm{QC}}(t-\Delta T)$. The differences $\delta s$ and $\delta \omega$ are very sensitive to the time offset $\Delta T$ applied to the QC run. In particular, at late times, when $s$ and $\omega$ vary rapidly, even a small change in $\Delta T$ causes the differences to deviate significantly from their expected oscillatory behaviour around zero. Looking at both $\delta s$ and $\delta \omega$, we estimate a time offset $\Delta T / M_{\mathrm{ADM}}=59 \pm 1$ between the QC and the F runs. 


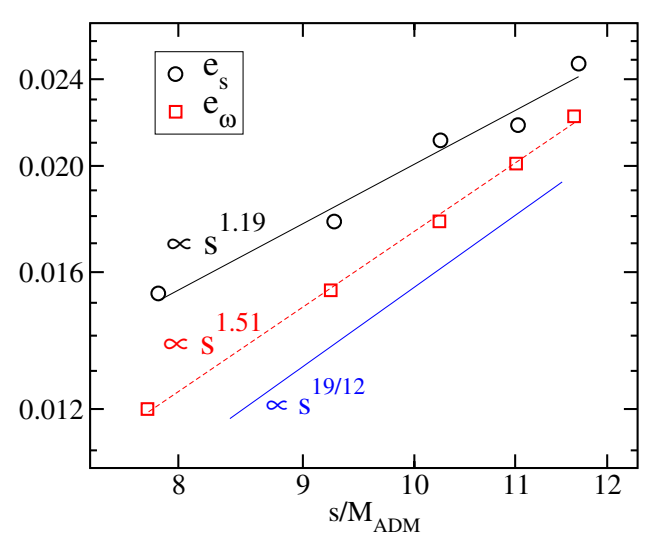

Figure 6. Orbital eccentricity of the QC evolution estimated from variations in proper separation, $e_{s}$, and from variations in orbital frequency, $e_{\omega}$. Also shown in this $\log -\log$ plot are best-fit power laws to each set of data, as well as the scaling predicted by Peters [8] with power $19 / 12 \approx 1.58$.

\subsection{Measuring eccentricity}

The evolution of the F initial data appears to have very low-orbital eccentricity, so it can be used as a reference from which the eccentricity of the QC run can be estimated. We can define an eccentricity for the $\mathrm{QC}$ evolution, for example, from the relative proper separation,

$$
e_{s}=\frac{|\delta s|}{s}
$$

where this equation is to be evaluated at the extrema of $\delta s$. Similarly, we can define a different measure of eccentricity from the variations in $\omega_{\text {orbit }}$ by evaluating

$$
e_{\omega}=\frac{|\delta \omega|}{2 \omega}
$$

at the extrema of $\delta \omega$. The factor of 2 in the definition of $e_{\omega}$ arises from angular momentum conservation, which makes the orbital frequency proportional to the square of the radius of the orbit. In Newtonian gravity, $e_{s}=e_{\omega}$ to first order in eccentricity. Since the F initial data result in a factor of 10 smaller oscillations in $\mathrm{d} s / \mathrm{d} t$ than the QC data, we expect these eccentricity estimates to be affected by the residual eccentricity of the $\mathrm{F}$ run at only the $10 \%$ level.

The orbital eccentricity of the QC run, estimated using equations (24) and (25), is plotted as a function of proper separation between the black holes in figure 6 . We see that these eccentricities decay during the inspiral, as expected. Within our estimated $10 \%$ errors, these eccentricities are consistent with a power law dependence on the proper separation, $e \propto s^{p}$. The eccentricity $e_{s}$ based on the proper separation is consistently somewhat larger than $e_{\omega}$, and it decays somewhat more slowly. Peters [8] derived the evolution of the orbital eccentricity during an inspiral due to the emission of gravitational waves using the quadrupole approximation. His result in the $e \ll 1$ limit predicts that $e \propto a^{19 / 12}$, where $a$ is the semi-major axis of the orbit and where the constant of proportionality depends on the initial conditions. Using $a \approx s / 2$, his formula predicts that the eccentricity should decay as

$$
e \propto s^{19 / 12} .
$$

Figure 6 confirms that $e_{\omega}$ follows this prediction quite closely, while $e_{s}$ has a somewhat smaller power law exponent. 
The eccentricities measured here are actually the relative eccentricities of the QC and the F orbits. The eccentricity of the QC run that we infer depends therefore on the residual eccentricity of the F run. A more intrinsic approach, used recently by Buonanno et al [27], is to fit some eccentricity-dependent quantity to a full cycle (or more) of the orbital data. This approach yields similar, but somewhat smaller, eccentricities than those found here (despite our use of a QC orbit having larger initial separation and so presumably smaller initial eccentricity).

\subsection{Waveform extraction}

We now turn our attention to the problem of extracting the gravitational wave signals from our numerical simulations using the Newman-Penrose quantity $\Psi_{4}$. Given a spatial hypersurface with timelike unit normal $n^{\mu}$, and given a spatial unit vector $r^{\mu}$ in the direction of wave propagation, the standard definition of $\Psi_{4}$ is the following component of the Weyl curvature tensor:

$$
\Psi_{4}=-C_{\alpha \mu \beta \nu} \ell^{\mu} \ell^{\nu} \bar{m}^{\alpha} \bar{m}^{\beta},
$$

where $\ell^{\mu} \equiv \frac{1}{\sqrt{2}}\left(n^{\mu}-r^{\mu}\right)$ and $m^{\mu}$ is a complex null vector (satisfying $m^{\mu} \bar{m}_{\mu}=1$ ) that is orthogonal to $r^{\mu}$ and $n^{\mu}$. Here an overbar denotes complex conjugation.

For (perturbations of) flat spacetime, $\Psi_{4}$ is typically evaluated on coordinate spheres, and in this case the usual choices for $r^{\mu}$ and $m^{\mu}$ are

$$
\begin{aligned}
r^{\mu} & =\left(\frac{\partial}{\partial r}\right)^{\mu}, \\
m^{\mu} & =\frac{1}{\sqrt{2} r}\left(\frac{\partial}{\partial \theta}+\mathrm{i} \frac{1}{\sin \theta} \frac{\partial}{\partial \phi}\right)^{\mu},
\end{aligned}
$$

where $(r, \theta, \phi)$ denote the standard spherical coordinates. With this choice, $\Psi_{4}$ can be expanded in terms of spin-weighted spherical harmonics of weight -2 :

$$
\Psi_{4}(t, r, \theta, \phi)=\sum_{l m} \Psi_{4}^{l m}(t, r)_{-2} Y_{l m}(\theta, \phi),
$$

where the $\Psi_{4}^{l m}$ are expansion coefficients defined by this equation.

For curved spacetime, there is considerable freedom in the choice of the vectors $r^{\mu}$ and $m^{\mu}$, and different researchers have made different choices [7, 27, 36-40] that are all equivalent in the $r \rightarrow \infty$ limit. We choose these vectors by first picking an extraction two-surface $\mathcal{E}$ that is a coordinate sphere $\left(r^{2}=x^{2}+y^{2}+z^{2}\right)$ centred on the centre of mass of the binary system (using the global asymptotically Cartesian coordinates employed in our code). We choose $r^{\mu}$ to be the outward-pointing spatial unit normal to $\mathcal{E}$ (that is, we choose $r_{i}$ proportional to $\nabla_{i} r$ ). Then we choose $m^{\mu}$ according to equation (28b), using the standard spherical coordinates $\theta$ and $\phi$ defined on these coordinate spheres. Finally, we use equations (27) and (29) to define the $\Psi_{4}^{l m}$ coefficients. Note that our $m^{\mu}$ is not exactly null nor exactly of unit magnitude at finite $r$, so our definition of $\Psi_{4}^{l m}$ will disagree with the waveforms observed at infinity (and with those computed by other groups). Our definition does, however, agree with the standard definition given in equations (27)-(29) as $r \rightarrow \infty$, so our definition only disagrees with the standard one by a factor of order $1+\mathcal{O}(1 / r)$. In this paper, we compute $\Psi_{4}^{l m}$ in the same way and at the same extraction radius for all runs, so the $\mathcal{O}(1 / r)$ effects should not significantly affect our comparisons of these waveforms.

Since our simulations use high spatial resolution all the way to the outer boundary, the outgoing radiation is fully resolved everywhere. Therefore, we could extract waveforms at 

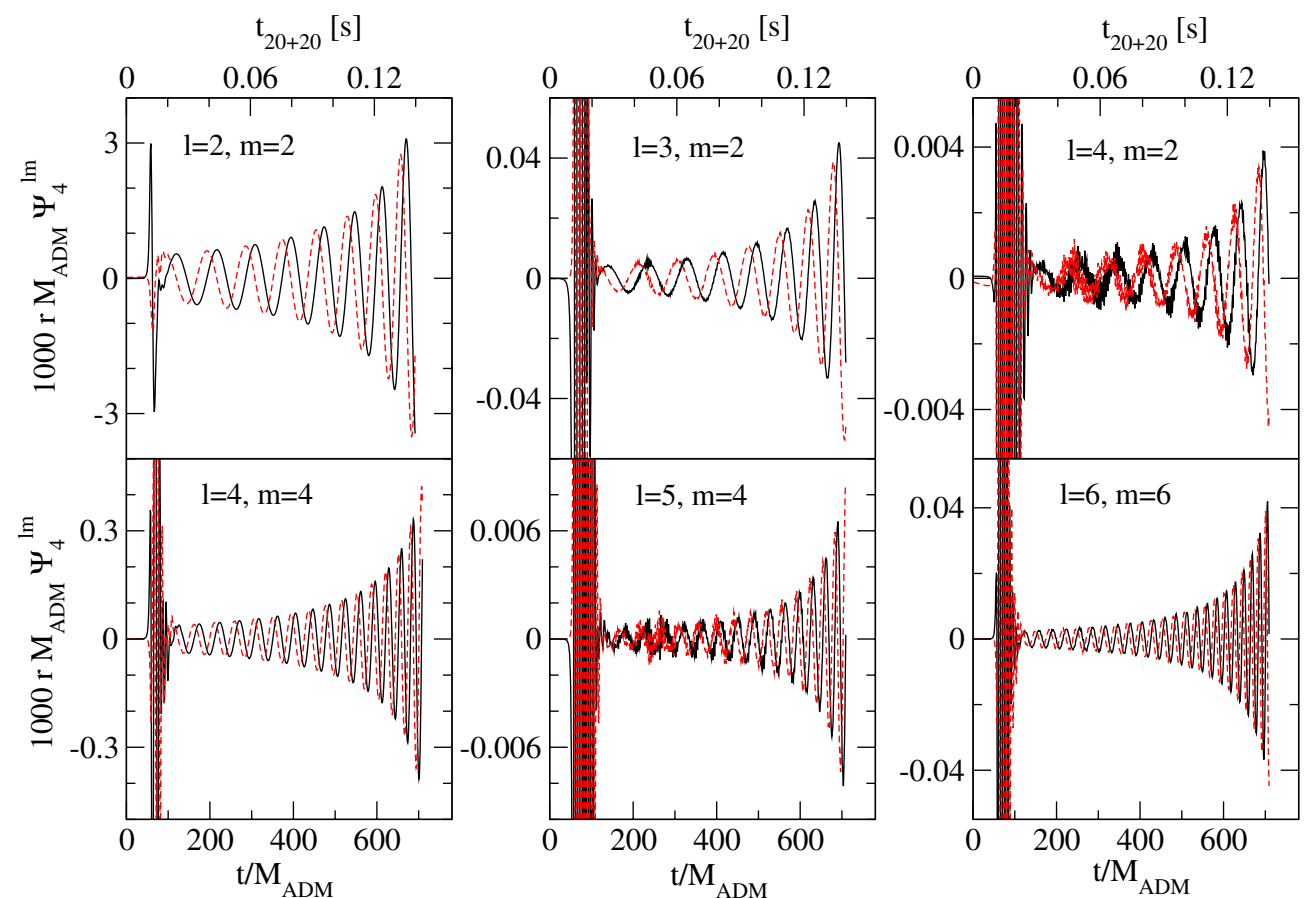

Figure 7. Waveforms for the F2 run. Plotted are the six dominant $\Psi_{4}^{l m}$ coefficients, scaled by the factor $1000 r M_{\mathrm{ADM}}$. Solid lines represent the real parts and dashed lines the imaginary parts of $\Psi_{4}^{l m}$. The time axes are labelled in geometric units at the bottom, and in SI units for a $20+20 M_{\odot}$ binary at the top.

very large radii. The extracted wave signal lags the dynamics of the binary by the light-travel time to the extraction radius, and our evolutions currently fail shortly before merger. So extracting the wave signal at a very large radius would miss the most interesting part of the waveform close to merger. In order to retain most of the signal, we compromise by extracting the radiation at an intermediate distance: $R / M_{\mathrm{ADM}}=57$. Figure 7 presents the dominant waveform coefficients $\Psi_{4}^{l m}$. The $\Psi_{4}^{44}$ coefficient is about a factor of 10 smaller than the largest coefficient, $\Psi_{4}^{22}$. The $\Psi_{4}^{32}$ and $\Psi_{4}^{66}$ coefficients are smaller by about another order of magnitude, and the $\Psi_{4}^{42}$ and $\Psi_{4}^{54}$ coefficients have amplitudes that are only about $\sim 1 / 1000$ that of $\Psi_{4}^{22}$.

\subsection{Waveform comparisons}

In this section, we make a number of quantitative comparisons between the waveforms produced by the evolution of quasi-circular, QC, initial data and those produced by the lower eccentricity, $\mathrm{F}$, initial data.

We can define a gravitational wave frequency associated with $\Psi_{4}^{l m}$ by writing

$$
\Psi_{4}^{l m}=A_{l m}(t) \mathrm{e}^{-\mathrm{i} \phi_{l m}(t)}
$$

where $A_{l m}(t)$ is its (real) amplitude and $\phi_{l m}(t)$ its (real) phase. The frequency, $\Omega_{l m}$, associated with $\Psi_{4}^{l m}$ is then defined as

$$
\Omega_{l m}=\frac{\mathrm{d} \phi_{l m}}{\mathrm{~d} t}
$$




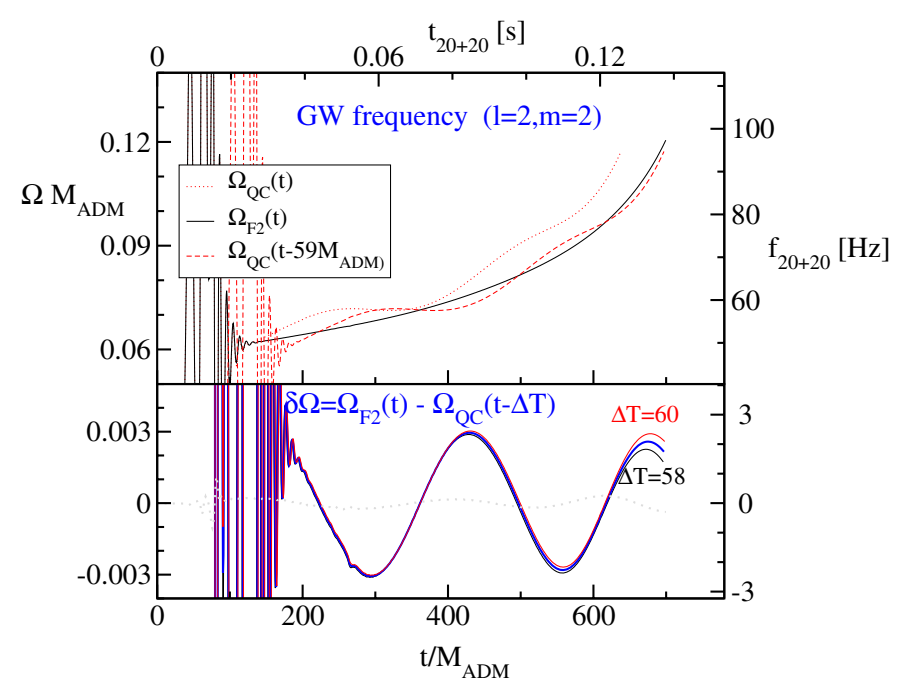

Figure 8. Frequency $\Omega_{22}$ of the gravitational waves extracted from the phase of $\Psi_{4}^{22}$. The left/bottom axes show geometric units, the right/top axes present SI units for a $20+20 M_{\odot}$ binary. The dotted line in the lower panel represents the difference between the E1 and F2 runs.

Figure 8 shows comparisons of the frequency of the dominant mode, $\Omega_{22}$, from the QC and the $\mathrm{F}$ runs. This figure confirms the basic picture that emerged from our discussion in sections 5.1 and 5.2: a time offset $\Delta t$ must be used to compare the QC and $\mathrm{F}$ runs properly; the QC run has an orbital eccentricity which causes $\Omega_{22}$ to oscillate, and these oscillations are largely absent from the $\mathrm{F}$ run. Indeed, apart from the factor of 2 difference between orbital and the gravitational wave frequencies, the top panel of figure 8 looks very much like figure 2. This indicates that our coordinates are very well behaved - a feature that has also been observed in other numerical simulations, e.g. [41].

In order to make more detailed comparisons between the QC and the $\mathrm{F}$ waveforms, a phase offset $\Delta \phi$ in addition to the time offset $\Delta T$ must be taken into account. These offsets are used then to redefine the waveform of the $\mathrm{QC}$ run

$$
\tilde{\Psi}_{4 \mathrm{QC}}^{l m}(t) \equiv \mathrm{e}^{-\mathrm{i} m \Delta \phi} \Psi_{4 \mathrm{QC}}^{l m}(t-\Delta T) .
$$

The same time and phase offsets are used for all values of $l$ and $m$. Note that $\Delta \phi$ and $\Delta T$ represent differences between the QC and $\mathrm{F}$ evolutions. These offsets differ therefore from those often used in LIGO data analysis, where offsets are used to set the time and orbital phase at which a binary signal enters the LIGO band at $40 \mathrm{~Hz}$.

We now estimate the phase offset $\Delta \phi$ needed in equation (32) to allow us to make direct comparisons between the QC and the F2 waveforms. We consider two effects. First, the orbital phase of the time-shifted QC run differs from that of the F2 run by the phase accumulated by the $\mathrm{F} 2$ run during the time $0 \leqslant t \leqslant \Delta T$. Second, the orbital frequencies of the $\mathrm{QC}$ and F2 runs differ, and this difference oscillates in time (cf the right panel of figure 5), so the orbital phase difference between the two runs also oscillates in time. We take both of these effects into account: first, we evaluate the time-dependent phase difference, $\Delta \phi(t)$, between the waveforms of the time offset QC run, $\Psi_{4 \mathrm{QC}}(t-\Delta t)$, and the F run, $\Psi_{4 \mathrm{~F}}(t)$; second, we evaluate the time average of this $\Delta \phi(t)$ to obtain $\Delta \phi \approx 1.83$. Using this value of $\Delta \phi$ leads to waveforms for the QC and F2 evolutions that agree as well as can be expected in the presence of the other systematic errors, described below. 

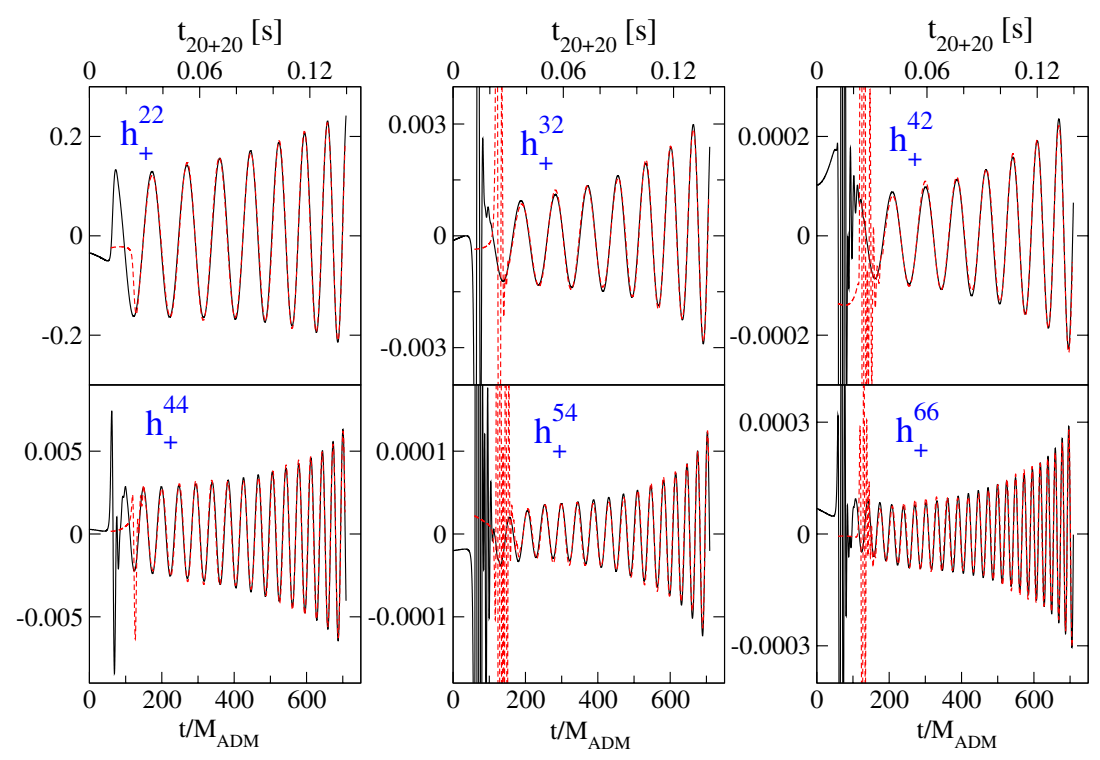

Figure 9. Waveforms $h_{+}^{l m}$ (normalized by $r / M_{\mathrm{ADM}}$ ) for the six dominant ${ }_{2} Y^{l m}$ modes. The solid lines represent evolution of the low-eccentricity initial data (run F2). The dashed lines represent evolution of QC initial data time shifted by $\Delta T=59 M_{\mathrm{ADM}}$ and phase rotated by $\Delta \phi=1.83$. The time axes are labelled in geometric units at the bottom and in SI units for a $20+20 M_{\odot}$ inspiral at the top.

The two gravitational wave polarizations, $h_{+}(t)$ and $h_{\times}(t)$, are the real functions related to $\Psi_{4}$ by

$$
\Psi_{4}=\ddot{h}_{+}-\mathrm{i} \ddot{h}_{\times} .
$$

Consequently, the ${ }_{-2} Y_{l m}$ components of $h_{+}(t)$ and $h_{\times}(t)$ can be obtained by the double time integral

$$
h_{+}^{l m}(t)-\mathrm{i} h_{\times}^{l m}(t)=\int_{t_{i}}^{t} \mathrm{~d} \tau \int_{t_{i}}^{\tau} \mathrm{d} \tau^{\prime} \Psi_{4}^{l m}\left(\tau^{\prime}\right)+C_{l m}+D_{l m} t .
$$

The constants $C_{l m}$ and $D_{l m}$ account for the (unknown) values of $h$ and $\dot{h}$ at the initial time $t_{i}$. If the full waveforms were known, they could be determined either at very early times or at very late times (i.e. after the merger and ringdown). Since we do not have complete waveforms for the present evolutions, we choose $C_{l m}$ and $D_{l m}$ that make the average and the first moment of $h_{+x}^{\operatorname{lm}}(t)$ vanish:

$$
\int_{t_{1}}^{t_{2}} \mathrm{~d} \tau h_{+\times}^{l m}(\tau)=0=\int_{t_{1}}^{t_{2}} \mathrm{~d} \tau \tau h_{+\times}^{l m}(\tau) .
$$

The integration interval $\left[t_{1}, t_{2}\right]=\left[160 M_{\mathrm{ADM}}, 706 M_{\mathrm{ADM}}\right]$ is chosen to be the largest interval (excluding the initial transient radiation burst) on which data are available for both runs.

Figure 9 shows the waveforms $h_{+}^{l m}$ for the evolution F2 (solid lines) and QC (dashed lines). To the eye, the waveforms look essentially identical. To quantify how well the two waveforms match, we use simple overlap integrals in the time domain:

$$
\mu=\frac{\left\langle h_{1}, h_{2}\right\rangle}{\left\|h_{1}\right\|\left\|h_{2}\right\|},
$$


Table 2. Waveform overlaps between the low-eccentricity run F2 and quasi-circular run QC (computed from runs with medium and high resolution). Each mode of QC has been time shifted and rotated by $\Delta T=59 M_{\mathrm{ADM}}$ and $\Delta \phi=1.83$. These numbers are subject to additional systematic effects as discussed in the text.

\begin{tabular}{llllll}
\hline & \multicolumn{2}{c}{ High resolution } & & \multicolumn{2}{c}{ Medium resolution } \\
\cline { 2 - 3 } Mode & $\mu\left(h_{+\mathrm{F}}^{l m}, \bar{h}_{\mathrm{QC}+}^{l m}\right)$ & $\mu\left(h_{\mathrm{F} \times}^{l m}, \bar{h}_{\mathrm{QC} \times}^{l m}\right)$ & & $\mu\left(h_{+\mathrm{F}}^{l m}, \bar{h}_{\mathrm{QC}+}^{l m}\right)$ & $\mu\left(h_{\mathrm{F} \times}^{l m}, \bar{h}_{\mathrm{QC} \times}^{l m}\right)$ \\
\hline$l=2, m=2$ & 0.998 & 0.998 & 0.998 & 0.998 \\
$l=3, m=2$ & 0.997 & 0.997 & 0.997 & 0.998 \\
$l=4, m=2$ & 0.996 & 0.997 & 0.996 & 0.998 \\
$l=4, m=4$ & 0.991 & 0.991 & 0.993 & 0.993 \\
$l=5, m=4$ & 0.987 & 0.979 & 0.983 & 0.982 \\
$l=6, m=6$ & 0.981 & 0.980 & 0.986 & 0.982 \\
\hline
\end{tabular}

where $\left\langle h_{1}, h_{2}\right\rangle \equiv \int_{t_{1}}^{t_{2}} \mathrm{~d} t h_{1}(t) h_{2}(t)$, and $\|h\|^{2} \equiv\langle h, h\rangle$. The quantity $\mu$ gives the loss of the signal-to-noise ratio obtained by filtering waveform $h_{1}$ with waveform $h_{2}$. We evaluate the overlap integral in the time domain, rather than the frequency domain, to allow us to truncate the waveforms easily to the interval $\left[t_{1}, t_{2}\right]$ during which both waveforms are available. During the evolutions presented here the gravitational wave frequency changes by only a factor of 2, so our decision not to weight by the LIGO noise spectrum should not change our results significantly for frequencies near the minimum of the noise curve. Furthermore, we evaluate $\mu$ directly for the different modes $h_{+, x}^{l m}$, rather than for specific observation directions. This allows us to compare differences in the higher order modes with smaller amplitudes, which would otherwise be swamped by the dominant $l=m=2$ mode.

The overlaps between the QC and the F2 waveforms, obtained at $\Delta T=59 M_{\mathrm{ADM}}$ and $\Delta \phi=1.83$, are summarized in table 2 . Both medium and high resolution overlaps are given in table 2, confirming that the overlaps are not dominated by numerical errors. We note, however, that the medium resolution runs have more noise in the higher order modes at early times; so we shortened the integration interval to $\left[t_{1}, t_{2}\right]=\left[200 M_{\mathrm{ADM}}, 706 M_{\mathrm{ADM}}\right]$ to avoid contamination in those waveforms.

The dominant uncertainty in the computed overlap $\mu$ arises because of our uncertainties in the integration constants $C_{l m}$ and $D_{l m}$ in equations (34) and (35). Because the waveform has finite length, these constants are known only to an accuracy of $\sim 1 / N_{\text {cyc }}$, where $N_{\text {cyc }}$ is the number of cycles in the waveform. This error depends only on the length of the evolution, and can only be reduced by longer evolutions, not by higher resolution evolutions. We show in appendix B (to lowest order in the uncertainties of $C_{l m}$ and $D_{l m}$ ) that the overlaps quoted in table 2 are upper bounds. We also derive lower bounds for these overlaps there, which are smaller than the values given in table 2 by about $12 /\left(\pi N_{\text {cyc }}\right)^{2}$. So these lower bounds are about 0.02 smaller than the table 2 values for the $m=2$ modes, and 0.002 smaller for the $m=6$ modes. This systematic uncertainty is much larger than the mismatch of the waveforms for the $m=2$ modes, so maximizing the overlaps by varying $\Delta T$ and $\Delta \phi$ as independent parameters is not justified.

\section{Discussion}

In this paper, we have extended the quasi-equilibrium initial-data formalism to binary black holes with nonzero radial velocities. We have also used this formalism to construct initial data whose evolution results in very low-eccentricity orbits: about an order of magnitude smaller than the orbits of quasi-circular initial data. 
The main differences between evolutions of the quasi-circular, QC, and the loweccentricity, F, initial data sets are overall time and phase shifts: the QC initial data represent the binary at a point closer to merger. When we correct for these shifts, the orbital trajectories of the black holes and the gravitational waveforms they produce agree very well between the two runs. Various parameters measured in the QC run (e.g. orbital frequency or proper separation) oscillate around the corresponding values from the $\mathrm{F}$ run. The gravitational wave phase oscillates as well, but no significant coherent phase difference builds up during the five orbits studied here. We find waveform overlaps between the high-eccentricity and low-eccentricity runs of about 0.99 . Therefore, it appears that for the last five orbits before merger the differences between quasi-circular and low-eccentricity initial data are not important for event detection in gravitational wave detectors. Longer evolutions (e.g. equal mass binaries starting at larger separation, as well as unequal mass binaries with a longer radiation reaction time scale) have more cycles during which phase shifts could in principle accumulate. However, orbital eccentricity tends to decay during an inspiral and the orbital eccentricity in quasi-circular data should decrease as the initial separation increases, so longer evolutions are probably less sensitive to the eccentricity in the initial data. Thus we anticipate that the eccentricity of quasi-circular initial data will not play a significant role when longer evolutions are used for event detection, but further study would be needed to confirm this.

Finally, we note that construction of low-eccentricity inspiral initial data may be more difficult when the black holes carry generic spin. The process described in this paper merely adjusts the orbital parameters to obtain a trajectory without oscillations on the orbital timescale. For non-spinning equal-mass black holes sufficiently far from merger, a non-oscillatory inspiral trajectory seems to be a reasonable choice. But if non-negligible spins are present, this is not likely to be the case. For spins that are not aligned with the orbital angular momentum, the approximate helical Killing vector is lost, and there are likely to be a variety of oscillations on the orbital time scale. In these cases, a more sophisticated model of the desired circularized orbit will be needed before a procedure for adjusting the orbital parameters to the appropriate values can be formulated.

\section{Acknowledgments}

We thank Gregory Cook for inspiring discussions, including the initial suggestion to add radial motion to quasi-circular initial data. We also thank Ilya Mandel for suggesting the comparison to Peters' calculation [8]. This work was supported in part by grants from the Sherman Fairchild Foundation to Caltech and Cornell, and from the Brinson Foundation to Caltech, by NSF grants PHY-0099568, PHY-0244906, PHY-0601459, DMS-0553302 and NASA grants NAG5-12834, NNG05GG52G at Caltech, and by NSF grants PHY-0312072, PHY-0354631, and NASA grant NNG05GG51G at Cornell. Some of the simulations discussed here were produced with LIGO Laboratory computing facilities. LIGO was constructed by the California Institute of Technology and Massachusetts Institute of Technology with funding from the National Science Foundation and operates under cooperative agreement PHY-0107417. This paper has been assigned LIGO document number LIGO-P060071-00-Z.

\section{Appendix A. Quasi-equilibrium initial data in inertial coordinates}

In this appendix we show that $\left(\psi_{\mathrm{co}}, \beta_{\mathrm{co}}^{i}-\xi_{\text {rot }}, \alpha_{\mathrm{co}}\right)$, where $\xi_{\text {rot }}^{i}=\left(\boldsymbol{\Omega}_{\mathbf{0}} \times \mathbf{r}\right)^{i}$, is a solution to the XCTS equations $(8 a)-(8 c)$ in asymptotically inertial coordinates (with appropriately modified boundary conditions) whenever $\left(\psi_{\mathrm{co}}, \beta_{\mathrm{co}}^{i}, \alpha_{\mathrm{co}}\right)$ is a solution in co-rotating coordinates. We 
also show that this solution leads to the same physical metric $\gamma_{i j}$ and extrinsic curvature $K_{i j}$ as the original solution in co-rotating coordinates. The proof relies on three key observations: first, both solutions are assumed to make the same choice of free data equations $(9 a)$ and $(9 b)$, $(15 a)$, and $(15 b)$; second, the shift enters the XCTS equations and the boundary conditions (almost) solely through the conformal Killing operator, $(\tilde{\mathbb{L}} \beta)^{i j}$, and third, $\xi_{\text {rot }}^{i}$ is a conformal Killing vector, so $\left(\tilde{\mathbb{L}} \xi_{\text {rot }}\right)^{i j}=0$. Hence the term $-\xi_{\text {rot }}^{i}$ that is added to $\beta_{\text {co }}^{i}$ (mostly) drops out of the equations.

We first show that the XCTS equations remain satisfied. Since $\left(\tilde{\mathbb{L}} \xi_{\text {rot }}\right)^{i j}=0$, it follows from equation (5) that $\tilde{A}_{i j}$ is unchanged by the addition of $\xi_{\text {rot }}^{i}$. So equations $(8 a)$ and $(8 b)$ remain satisfied. The only other shift containing term in equation $(8 c)$ is $\beta^{i} \partial_{i} K$, which vanishes because $\partial_{i} K=0$ from the choice of free data $(K=0)$ in equation $(15 b)$, so equation $(8 c)$ also remains satisfied.

We turn next to the boundary conditions. The boundary conditions used for the corotating coordinate representation of the XCTS equations are equations $(9 c)-(9 g)$ and $(15 c)$, while those used for the inertial frame representation are the same, except equations $(9 d)$ and $(9 e)$ are replaced by equations $(16 a)$ and $(16 b)$. The boundary conditions, equations $(9 c)$ and (15c), depend only on $\psi$ and $\alpha$ and therefore remain satisfied. The apparent horizon boundary condition, equation $(9 f)$, implies the boundary condition on the conformal factor equation (13), which is unchanged since $\left(\tilde{\mathbb{L}} \xi_{\text {rot }}\right)^{i j}=0$, and the new outer boundary condition, equation (16a), also holds because $\beta_{\mathrm{co}}^{i}$ satisfies equation ( $\left.9 d\right)$.

The only remaining boundary conditions then are equations $(16 b)$ and $(9 g)$. Because $\theta=0$ and $\sigma_{i j}=0$, the null surface generated by $k^{\mu}$ coincides with the world tube of the apparent horizons, $\mathcal{S}_{\mathrm{AH}}$. The normal to this null surface is $k^{\mu}$, because $k^{\mu}$ is normal to $\mathcal{S}$ by construction, and because $k^{\mu} k_{\mu}=0$. Therefore, in order for $\partial_{t}+\xi_{\text {rot }}^{i} \partial_{i}$ to be tangent to $\mathcal{S}_{\mathrm{AH}}$, as required by the boundary condition equation $(16 b)$, it must be orthogonal to $k^{\mu}$. The vector $\partial_{t}+\xi_{\text {rot }}^{i} \partial_{i}$ has components $\alpha n^{\mu}+\beta^{\mu}+\xi_{\text {rot }}^{\mu}$, where $\beta^{\mu}=\left[0, \beta^{i}\right]$ and $\xi_{\text {rot }}^{\mu}=\left[0, \xi_{\text {rot }}^{i}\right]$. Using $k^{\mu}=\left(n^{\mu}+s^{\mu}\right) / \sqrt{2}$, it follows that

$$
0=\left(\partial_{t}+\xi_{\text {rot }}^{i} \partial_{i}\right) \cdot k=\frac{1}{\sqrt{2}}\left[-\alpha+\left(\beta^{i}+\xi_{\text {rot }}^{i}\right) s_{i}\right] .
$$

This condition implies

$$
\beta^{i}=\alpha s^{i}-\xi_{\text {rot }}^{i}+\zeta^{i} \text { on } \mathcal{S}
$$

with $\zeta^{i} s_{i}=0$, i.e., equation (17) in the main text. So the boundary condition equation (16b) is satisfied because $\beta_{\mathrm{co}}^{i}=\alpha s^{i}+\zeta^{i}$ satisfies equation (12).

The vector $\zeta^{i}$ that appears in equation (A.2) is further constrained by the shear boundary condition, equation $(9 g)$, which we consider next. The shear $\sigma_{i j}$ is defined as

$$
\sigma_{\mu \nu}=\perp_{\mu \nu}{ }^{\rho \sigma(4)} \nabla_{\rho} k_{\sigma}
$$

where $\perp_{\mu \nu}{ }^{\rho \sigma}=h_{\mu}{ }^{(\rho} h_{\nu}{ }^{\sigma)}-\frac{1}{2} h_{\mu \nu} h^{\rho \sigma}$. Substituting equation (11) into this expression, and subsequently using equations (2), (4), and (5) results in

$$
\sqrt{2} \sigma_{i j}=-\frac{1}{2 \alpha} \perp_{i j}{ }^{k l}\left[(\mathbb{L} \beta)_{k l}-\psi^{4} \tilde{u}_{k l}\right]+\perp_{i j}{ }^{k l} \nabla_{k} s_{l} .
$$

For any vector field $v^{i}$ decomposed into normal and tangential parts, $v^{i}=v^{m} s_{m} s^{i}+v_{\|}^{i}$, it follows that

$$
\perp_{i j}{ }^{k l}(\mathbb{L} v)_{k l}=\left(\mathbb{L}_{\mathcal{S}} v_{\|}\right)_{i j}+2 v^{m} s_{m} \perp_{i j}{ }^{k l} \nabla_{k} s_{l} .
$$

Using this identity and equation (17), the shear can be rewritten as

$$
\sqrt{2} \sigma_{i j}=\frac{1}{2 \alpha} \perp_{i j}{ }^{k l}\left[\left(\mathbb{L} \xi_{\text {rot }}\right)_{k l}+\psi^{4} \tilde{u}_{k l}\right]-\frac{1}{2 \alpha}\left(\mathbb{L}_{\mathcal{S}} \zeta\right)_{i j} .
$$


Once more, $\xi_{\text {rot }}^{i}$ drops out because it is a conformal Killing vector. Also, since $\tilde{u}_{i j}=0$ by equation $(9 a)$, we find that the shear vanishes iff $\zeta^{i}$ is a conformal Killing vector within the 2-surface $\mathcal{S}$ :

$$
\sigma_{i j}=0 \quad \Leftrightarrow \quad 0=\left(\mathbb{L}_{\mathcal{S}} \zeta\right)^{i j}
$$

Equation (18) now follows from the identity $\left(\mathbb{L}_{\mathcal{S}} \zeta\right)^{i j}=\psi^{-4}\left(\tilde{\mathbb{L}}_{\mathcal{S}} \zeta\right)^{i j}$. This implies then that the boundary condition equation $(9 g)$ is satisfied since it is assumed to be satisfied in the co-rotating case.

Finally, we note that the physical metric $\gamma_{i j}$ and extrinsic curvature $K_{i j}$ produced by the inertial frame version of the problem are identical to those of the original co-rotating frame version. The conformal metric and conformal factor are identical in the two versions, so the physical metrics are identical trivially from equation (3). Since $\xi_{\text {rot }}^{i}$ is a conformal Killing vector, it follows that $A_{i j}$ is identical from equation (5); so it follows from equation (4) (with $K=0$ ) that the extrinsic curvatures are identical as well.

\section{Appendix B. Errors caused by finite-length waveforms}

The error in the waveform overlaps caused by the uncertainty in the integration constants can be determined as follows. Denote our numerically computed waveforms by $h_{x}+\varepsilon_{x}$, where $h_{x}$ stands for the unknown 'true' waveform obtained with the correct values of the integration constants, and $\varepsilon_{x}$ represents the error introduced by computing these constants with a truncated waveform. The label $x$ stands for either $\mathrm{F}$ or QC.

The quantity of interest is the overlap between the 'true' waveforms

$$
\mu\left(h_{\mathrm{F}}, h_{\mathrm{QC}}\right)=\frac{\left\langle h_{\mathrm{F}}, h_{\mathrm{QC}}\right\rangle}{\left\|h_{\mathrm{F}}\right\|\left\|h_{\mathrm{QC}}\right\|},
$$

where $\left\langle h_{1}, h_{2}\right\rangle \equiv \int_{t_{1}}^{t_{2}} h_{1}(t) h_{2}(t) \mathrm{d} t$, and $\|h\|^{2} \equiv\langle h, h\rangle$. The errors $\varepsilon_{x}$ are those caused by the uncertainty in the constants $C_{l m}$ and $D_{l m}$ in equation (34), and the $\varepsilon_{x}$ are therefore linear functions of time. Furthermore, choosing the integration constants by equation (35) makes the numerical waveforms $h_{x}+\varepsilon_{x}$ orthogonal to functions linear in time, so that $\left\langle h_{x}+\varepsilon_{x}, \varepsilon_{y}\right\rangle=0$, where $x, y \in\{F, Q C\}$. Using this result, and neglecting terms of order $\mathcal{O}\left(\varepsilon^{3}\right)$, one finds

$\mu\left(h_{\mathrm{F}}+\varepsilon_{\mathrm{F}}, h_{\mathrm{QC}}+\varepsilon_{\mathrm{QC}}\right)=\mu\left(h_{\mathrm{F}}, h_{\mathrm{QC}}\right)+\mu\left(h_{\mathrm{F}}, h_{\mathrm{QC}}\right)\left(\frac{\left\|\varepsilon_{\mathrm{F}}\right\|^{2}}{2\left\|h_{\mathrm{F}}\right\|^{2}}+\frac{\left\|\varepsilon_{\mathrm{QC}}\right\|^{2}}{2\left\|h_{\mathrm{QC}}\right\|^{2}}-\frac{\left\langle\varepsilon_{\mathrm{F}}, \varepsilon_{\mathrm{QC}}\right\rangle}{\left\|h_{\mathrm{F}}\right\|\left\|h_{\mathrm{QC}}\right\|}\right)$.

It is straightforward to show that $\mu\left(h_{\mathrm{F}}, h_{\mathrm{QC}}\right)=1-\mathcal{O}\left(\delta h^{2}\right)$, where $\delta h=h_{\mathrm{F}}-h_{\mathrm{QC}}$. Therefore, replacing $\mu\left(h_{\mathrm{F}}, h_{\mathrm{QC}}\right) \rightarrow 1$ in the last term of equation (B.2) changes the result only by terms of order $\mathcal{O}\left(\delta h^{2} \varepsilon_{x}^{2}\right)$. Furthermore, replacing $\left\|h_{\mathrm{QC}}\right\| \rightarrow\left\|h_{\mathrm{F}}\right\|$ in the denominators of equation (B.2) affects the result only by terms of order $\mathcal{O}\left(\delta h \varepsilon^{2}\right)$. Neglecting both of these higher order contributions, we find

$$
\mu\left(h_{\mathrm{F}}+\varepsilon_{\mathrm{F}}, h_{\mathrm{QC}}+\varepsilon_{\mathrm{QC}}\right)=\mu\left(h_{\mathrm{F}}, h_{\mathrm{QC}}\right)+\frac{\left\|\varepsilon_{\mathrm{F}}-\varepsilon_{\mathrm{QC}}\right\|^{2}}{2\left\|h_{\mathrm{F}}\right\|^{2}} .
$$

Because the last term is non-negative, the 'true' overlap $\mu\left(h_{\mathrm{F}}, h_{\mathrm{QC}}\right)$ is always smaller than the numerically computed overlap $\mu\left(h_{\mathrm{F}}+\varepsilon_{\mathrm{F}}, h_{\mathrm{QC}}+\varepsilon_{\mathrm{QC}}\right)$. Using the triangle inequality, we can bound the last term in equation (B.3) by the error $\left\|\varepsilon_{x}\right\|^{2} /\left\|h_{x}\right\|^{2}$ in either the $\mathrm{F}$ or the QC waveform:

$$
\frac{\left\|\varepsilon_{\mathrm{F}}-\varepsilon_{Q}\right\|^{2}}{2\left\|h_{\mathrm{F}}\right\|^{2}} \leqslant \frac{\left(\left\|\varepsilon_{\mathrm{F}}\right\|+\left\|\varepsilon_{\mathrm{QC}}\right\|\right)^{2}}{2\left\|h_{\mathrm{F}}\right\|^{2}} \approx 2 \frac{\left\|\varepsilon_{x}\right\|^{2}}{\left\|h_{x}\right\|}
$$


Finally, we estimate $\left\|\varepsilon_{x}\right\|^{2} /\left\|h_{x}\right\|^{2}$ by applying equations (34) and (35) to a pure sine-wave: $h(t)=\sin (t)$. It is straightforward to evaluate the integrals in equation (35) for this simple case, giving the bound $\|\varepsilon\|^{2} /\|h\|^{2} \leqslant 6 /\left(\pi N_{\text {cyc }}\right)^{2}$, where $N_{\text {cyc }}=\left(t_{2}-t_{1}\right) /(2 \pi)$ is the number of cycles in the interval $\left[t_{1}, t_{2}\right]$. Therefore, we arrive at the bounds

$\mu\left(h_{\mathrm{F}}+\varepsilon_{\mathrm{F}}, h_{\mathrm{QC}}+\varepsilon_{\mathrm{QC}}\right) \geqslant \mu\left(h_{\mathrm{F}}, h_{\mathrm{QC}}\right) \gtrsim \mu\left(h_{\mathrm{F}}+\varepsilon_{\mathrm{F}}, h_{\mathrm{QC}}+\varepsilon_{\mathrm{QC}}\right)-\frac{12}{\pi^{2} N_{\mathrm{cyc}}^{2}}$,

as mentioned in the main text.

\section{References}

[1] Barish B C and Weiss R 1999 LIGO and the detection of gravitational waves Phys. Today 52 44-50

[2] Acernese F et al 2002 The present status of the VIRGO central interferometer Class. Quantum Grav. 191421

[3] Pretorius F 2005 Evolution of binary black hole spacetimes Phys. Rev. Lett. 95121101

[4] Baker J G, Centrella J, Choi D-I, Koppitz M and van Meter J 2006 Gravitational wave extraction from an inspiralling configuration of merging black holes Phys. Rev. Lett. 96111102

[5] Campanelli M, Lousto C O, Marronetti P and Zlochower Y 2006 Accurate evolutions of orbiting black-hole binaries without excision Phys. Rev. Lett. 96111101

[6] Scheel M A, Pfeiffer H P, Lindblom L, Kidder L E, Rinne O and Teukolsky S A 2006 Solving Einstein's equations with dual coordinate frames Phys. Rev. D 74104006

[7] Brügmann B, Gonzalez J A, Hannam M, Husa S, Sperhake U and Tichy W 2006 Calibration of moving puncture simulations Preprint gr-qc/0610128

[8] Peters P C 1964 Gravitational radiation and the motion of two point masses Phys. Rev. 136 1224-32

[9] Cook G B 1994 Three-dimensional initial data for the collision of two black holes: II. Quasicircular orbits for equal-mass black holes Phys. Rev. D 50 5025-32

[10] Baumgarte T W 2000 The innermost stable circular orbit of binary black holes Phys. Rev. D 62024018

[11] Gourgoulhon E, Grandclément P and Bonazzola S 2002 Binary black holes in circular orbits: I. A global spacetime approach Phys. Rev. D 65044020

[12] Grandclément P, Gourgoulhon E and Bonazzola S 2002 Binary black holes in circular orbits: II. Numerical methods and first results Phys. Rev. D 65044021

[13] Baker B D 2002 Binary black holes in quasi-stationary circular orbits Preprint gr-qc/0205082v1

[14] Cook G B 2002 Corotating and irrotational binary black holes in quasi-circular orbits Phys. Rev. D 65084003

[15] Tichy W, Brügmann B, Campanelli M and Diener P 2003 Binary black hole initial data for numerical general relativity based on post-Newtonian data Phys. Rev. D 68064008

[16] Pfeiffer H P 2003 Initial data for black hole evolutions PhD Thesis Cornell University

[17] Cook G B and Pfeiffer H P 2004 Excision boundary conditions for black hole initial data Phys. Rev. D 70104016

[18] Yo H-J, Cook J N, Shapiro S L and Baumgarte T W 2004 Quasi-equilibrium binary black hole initial data for dynamical evolutions Phys. Rev. D 70084033

Yo H-J, Cook J N, Shapiro S L and Baumgarte T W 2004 Quasi-equilibrium binary black hole initial data for dynamical evolutions Phys. Rev. D 70089904 (erratum)

[19] Tichy W and Brügmann B 2004 Quasiequilibrium binary black hole sequences for puncture data derived from helical Killing vector conditions Phys. Rev. D 69024006

[20] Caudill M, Cook G B, Grigsby J D and Pfeiffer H P 2006 Circular orbits and spin in black-hole initial data Phys. Rev. D 74064011

[21] Dennison K A, Baumgarte T W and Pfeiffer H P 2006 Approximate initial data for binary black holes Phys. Rev. D 74064016

[22] Yunes N, Tichy W, Owen B J and Bruegmann B 2006 Binary black hole initial data from matched asymptotic expansions Phys. Rev. D 74104011

[23] Yunes N and Tichy W 2006 Improved initial data for black hole binaries by asymptotic matching of postNewtonian and perturbed black hole solutions Phys. Rev. D 74064013

[24] Baker J, Campanelli M, Lousto C O and Takahashi R 2002 Modeling gravitational radiation from coalescing binary black holes Phys. Rev. D 65124012

[25] Brandt S and Brügmann B 1997 A simple construction of initial data for multiple black holes Phys. Rev. Lett. 78 3606-9

[26] Miller M 2004 Circular orbit approximation for binary compact objects in general relativity Phys. Rev. D 69124013 
[27] Buonanno A, Cook G B and Pretorius F 2006 Inspiral, merger and ring-down of equal-mass black-hole binaries Preprint gr-qc/0610122

[28] York J W Jr 1999 Conformal 'thin sandwich' data for the initial-value problem of general relativity Phys. Rev. Lett. 82 1350-3

[29] Pfeiffer H P and York J W 2003 Extrinsic curvature and the Einstein constraints Phys. Rev. D 67044022

[30] Rieth R 1997 On the validity of Wilson's approach to general relativity Mathematics of Gravitation. Part II. Gravitational Wave Detection ed A Królak (Warsaw: Polish Academy of Sciences, Institute of Mathematics) pp $71-4$

[31] Pfeiffer H P, Kidder L E, Scheel M A and Teukolsky S A 2003 A multidomain spectral method for solving elliptic equations Comput. Phys. Commun. 152 253-73

[32] Lindblom L, Scheel M A, Kidder L E, Owen R and Rinne O 2006 A new generalized harmonic evolution system Class. Quantum Grav. 23 S447-62

[33] Friedrich H 1985 On the hyperbolicity of Einstein's and other gauge field equations Commun. Math. Phys. 100 525-43

[34] Pretorius F 2005 Numerical relativity using a generalized harmonic decomposition Class. Quantum Grav. 22 425-52

[35] Rinne O, Lindblom L and Scheel M A 2007 Testing outer boundary treatments for the Einstein equations Preprint gr-qc/0704:0782

[36] Fiske D R, Baker J G, van Meter J R, Choi D-I and Centrella J M 2005 Wave zone extraction of gravitational radiation in three-dimensional numerical relativity Phys. Rev. D 71104036

[37] Beetle C, Bruni M, Burko L M and Nerozzi A 2005 Towards a novel wave-extraction method for numerical relativity: I. Foundations and initial-value formulation Phys. Rev. D 72024013

[38] Nerozzi A, Beetle C, Bruni M, Burko L M and Pollney D 2005 Towards wave extraction in numerical relativity: the quasi-Kinnersley frame Phys. Rev. D 72024014

[39] Burko L M, Baumgarte T W and Beetle C 2006 Towards a wave-extraction method for numerical relativity: III. Analytical examples for the Beetle-Burko radiation scalar Phys. Rev. D 73024002

[40] Campanelli M, Kelly B J and Lousto C O 2006 The Lazarus project: II. Spacelike extraction with the quasiKinnersley tetrad Phys. Rev. D 73064005

[41] Pretorius F 2006 Simulation of binary black hole spacetimes with a harmonic evolution scheme Class. Quantum Grav. 23 S529-52 